\title{
AN ITERATIVE ALGORITHM FOR SPLIT EQUALITY FIXED POINT PROBLEM OF MULTIVALUED LIPSCHITZIAN QUASI-PSEUDOCONTRACTIVE MAPPINGS WITH APPLICATIONS
}

\author{
U. S. JIM ${ }^{1, *}$ AND D. I. IGBOKWE ${ }^{2}$
}

\begin{abstract}
We introduce an iterative algorithm for split equality fixed point and null point problem for multivalued Lipschitzian quasi-pseudocontractive mappings and maximal monotone operators which includes split equality feasibility problem, split equality problem, Split equality null point problem and other problem related to fixed point problems. Moreover, we establish a strong convergence results in real Hilbert spaces under some suitable conditions and reduce our main result to above-mentioned problems. Finally, we apply the study to split equality feasibility problem (SEFP), split equality equilibrium problem (SEEP), split equality variational inequality problem (SEVIP) and split equality optimization problem (SEOP). The results presented in the paper extend and improve many recent results.
\end{abstract}

\section{INTRODUCTION}

Let $C$ and $Q$ be closed and convex subsets of real Hilbert spaces $H_{1}$ and $H_{2}$, respectively. Consider two bounded linear operators $A: H_{1} \rightarrow H_{3}$ and $B: H_{2} \rightarrow H_{3}$, where $H_{3}$ is another real Hilbert space. The split equality feasibility problem consists of finding two points $x \in C$ and $y \in Q$ such that $A x=B y$. Split equality fixed problem allows asymmetric and partial relations between the variables $x$ and $y$, and covers many problems such as decomposition methods for partial differential equations, applications in game theory, and intensity-modulated radiation therapy. These broad applications caught the attention of many researchers, and eventually leading to various research output for the split equality feasibility problem, see for example [1, 2, 22, 32-34, 36].

Let $T: C \rightarrow 2^{C}$ be a multivalued mapping. An element $p \in C$ is called a fixed point of $T$ if $p \in T p$. The set of all fixed points of $T$ is denoted by $F(T)$. We say that $T$ satisfies the endpoint condition if $T p=p$ for all $p \in F(T)$.

A subset $D$ of $C$ is said to be proximal if for each $x \in C$, there exists $y \in D$ such that

$$
\|x-y\|=d(x, D)
$$

\footnotetext{
${ }^{1}$ Department of Mathematics, University of Uyo, P. M. B. 1017, Uyo - Nigeria

${ }^{2}$ Department of Mathematics, Michael Okpara University of Agriculture, Umudike, Abia StATE - NigERia

*Corresponding AUTHOR

E-mail addresses: ukojim@uniuyo.edu.ng, igbokwedi@yahoo.com.

Key words and phrases. split equality fixed point problem; quasi-pseudo-contractive mapping; maximal monotone operators; Halpern-Ishikawa type algorithm.
}

Received 01/09/2021. 
We denote by $C B(C), K(C)$, and $P(C)$ the families of all nonempty closed bounded subsets of $C$, nonempty compact subsets of $C$, and nonempty proximal bounded subsets of $C$, respectively. The Pompeiu-Hausdorff metric on $C B(C)$ is defined by

$$
H(A, B)=\max \left\{\sup _{a \in A} d(a, B), \sup _{b \in B} d(b, A)\right\}
$$

for all $A, B \in C B(C)$.

Lemma 1.1 ([28]) Let $K$ be a normed space. Let $T: K \rightarrow P(K)$ be a multivalued mapping and $P_{T}(x)=\{y \in T x:\|x-y\|=d(x, T x)\}$. Then the following are equivalent:

(i) $x \in T x$,

(ii) $P_{T} x=\{x\}$

(iii) $x \in F\left(P_{T}\right)$

moreover $F(T)=F\left(P_{T}\right)$.

Definition 1.1. Let $K$ be a nonempty closed convex subset of $H$.

A multivalued mapping $T: K \rightarrow C B(K)$ is said to be nonexpansive if

$$
H(T x, T y) \leq\|x-y\| \text { for all } x, y \in K
$$

A multivalued mapping $T: K \rightarrow C B(K)$ is said to be quasi-nonexpansive if $F(T) \neq \emptyset$ and

$$
H(T x, T p) \leq\|x-p\| \text { for all } x \in K, p \in F(T)
$$

A multivalued mapping $T: K \rightarrow C B(K)$ is said to be $k$-strickly pseudocontractive ([17]) if there exists $k \in(0,1)$ such that for all $x, y \in D(T)$ one has

$$
H^{2}(T x, T y) \leq\|x-y\|^{2}+k\|x-u-(y-v)\|^{2} \quad \forall u \in T x, v \in T y
$$

A multivalued mapping $T: K \rightarrow C B(K)$ is said to be pseudocontractive ([17]) if

$$
H^{2}(T x, T y) \leq\|x-y\|^{2}+\|x-u-(y-v)\|^{2} \quad \forall u \in T x, v \in T y
$$

A multivalued mapping $T: K \rightarrow C B(K)$ is said to be demicontractive [18, 20] if $F(T) \neq \emptyset$ and there exists $k \in[0,1)$ such that

$$
H^{2}(T x, T p) \leq\|x-p\|^{2}+k d^{2}(x, T x) \text { for all } x \in K, p \in F(T)
$$

A multivalued mapping $T: K \rightarrow C B(K)$ is said to be quasi-pseudocontractive ([20]) if $F(T) \neq \emptyset$ such that

$$
H^{2}(T x, T p) \leq\|x-p\|^{2}+d^{2}(x, T x) \text { for all } x \in K, p \in F(T)
$$

The following example shows that the class of demicontractive mapping is properly contained in the class of quasi-pseudocontractive mappings (see [20]).

Example 1.3 Let $X=\mathbb{R}$ (the reals with the usual metric). Define $T: \mathbb{R} \rightarrow 2^{\mathbb{R}}$ by

$$
T x=\left\{\begin{array}{lc}
{[-\sqrt{2} x, 0],} & x \in[0, \infty), \\
{[0,-\sqrt{2} x]} & x \in(-\infty, 0) .
\end{array}\right.
$$

Then $F(T)=\{0\}$. For each $x \in(-\infty, 0) \cup x \in(0, \infty)$,

$$
\begin{aligned}
H^{2}(T x, T 0) & =|\sqrt{2} x-0|^{2}=2|x-0|^{2}=|x-0|^{2}+|x-0|^{2}, \\
d^{2}(x, T x) & =|x-T 0|^{2}=|x-0|^{2} .
\end{aligned}
$$


therefore,

$$
\begin{aligned}
H^{2}(T x, T 0)=|x-0|^{2}+|x-0|^{2} & =|x-0|^{2}+d^{2}(x, T x) \\
& >|x-0|^{2}+k d^{2}(x, T x),
\end{aligned}
$$

for all $x \in \mathbb{R}$ and $\forall k \in(0,1)$. Therefore $T$ is quasi pseudocontractive.

Example 1.4 Let $X$ be a normed space. suppose $T$ is multivalued mapping such that $F(T) \neq \emptyset$ and $P_{T}$ is pseudocontractive-type mapping, then $P_{T}$ is quasi pseudocontractive.

Example 1.5 Let $X$ be a normed space. Let $T: D(T) \subseteq X \rightarrow P(X)$ be a multivalued pseudocontractive-type with nonempty fixed point set. Suppose $T p=\{p\}$ for all $p \in F(T)$, then for any $x \in D(T), p \in F(T)$ and $u \in T x$ with $\|x-u\|=d(x, T x)$ we have

$$
H^{2}(T x, T p)=|x-p|^{2}+|x-u|^{2}=|x-p|^{2}+d^{2}(x, T x)
$$

Remark 1.6 We easily observe that the class of quasi pseudocontractive operators includes the class of operators defined in equations (??) - (??).

Let $H_{1}$ and $H_{2}$ be real Hilbert spaces and $\mathrm{C}$ and $\mathrm{Q}$ be nonempty closed and convex subsets of $H_{1}$ and $H_{1}$ respectively. The split feasibility problem (SFP) is formulated as: to find

$$
x^{*} \in C \text { such that } A x^{*} \in Q
$$

where $A: H_{1} \rightarrow H_{2}$ is a bounded linear operator. In 1994, Censor and Elfving [9] first introduced the SFP in finite-dimensional Hilbert spaces for modeling inverse problems which arise fromphase retrievals and in medical image reconstruction [7]. It has been found that the SFP can also be used in various disciplines such as image restoration, computer tomography, and radiation therapy treatment planning $[8,10,11]$. The SFP in an infinite-dimensional real Hilbert space can be found in [7,10,12,13,31,33,36].

Moudafi [23-25] introduced the following split equality feasibility problem (SEFP) to find:

$$
x \in C, y \in Q \text { such that } A x=B y,
$$

where $A: H_{1} \rightarrow H_{3}$ and $B: H_{2} \rightarrow H_{3}$ are two bounded linear operators. Obviously, if $B=I$ (identity mapping on $H_{2}$ ) and $H_{3}=H_{2}$, then (13) reduces to (12).

In order to solve split equality feasibility problem (13), Moudafi [23] introduced the following simultaneous iterative method:

$$
\begin{aligned}
& x_{n+1}=P_{C}\left(x_{n}-\gamma A^{*}\left(A x_{n}-B y_{n}\right)\right) \\
& y_{n+1}=P_{Q}\left(y_{n}+\beta B^{*}\left(A x_{n}-B y_{n}\right)\right)
\end{aligned} n \in \mathbb{N} .
$$

and under suitable conditions, he proved the weak convergence of the sequence $\left\{\left(x_{n}, y_{n}\right)\right\}$ to a solution of (13) in Hilbert spaces.

In order to avoid using the projection, recently, Moudafi [13] introduced and studied the following problem: Let $T: H_{1} \rightarrow H_{1}$ and $S: H_{2} \rightarrow H_{2}$ be nonlinear operators such that $F(T) \neq \emptyset$ and $F(S) \neq \emptyset$, where $F(T)$ and $F(S)$ denote the sets of fixed points of $T$ and $S$ respectively. If $C=F(T)$ and $Q=F(S)$, then split equality feasibility problem (13) reduces to

$$
x \in F i x(T), y \in F i x(s) \text { such that } A x=B y,
$$

which is called a split equality fixed point problem (SEFPP). 
Moudafi [24] proposed the following iterative algorithm for finding a solution of SEFPP (15):

$$
\begin{aligned}
& x_{n+1}=T\left(x_{n}-\gamma_{n} A^{*}\left(A x_{n}-B y_{n}\right)\right) \\
& y_{n+1}=S\left(y_{n}+\beta_{n} B^{*}\left(A x_{n}-B y_{n}\right)\right)
\end{aligned} n \in \mathbb{N} .
$$

He also studied the weak convergence of the sequences generated by scheme (16) under the condition that $T$ and $S$ are firmly quasi-nonexpansive mappings.

Che and $\mathrm{Li}$ [16] proposed the following iterative algorithm for finding a solution of SEFPP (13):

$$
\begin{aligned}
u_{n} & =x_{n}-\gamma_{n} A^{*}\left(A x_{n}-B y_{n}\right) \\
x_{n+1} & =\alpha_{n} x_{n}+\left(1-\alpha_{n}\right) T u_{n} \\
v_{n} & =y_{n}+\beta_{n} B^{*}\left(A x_{n}-B y_{n}\right) \\
y_{n+1} & =\alpha_{n} y_{n}+\left(1-\alpha_{n}\right) S v_{n}
\end{aligned}
$$

They also established the weak convergence of the scheme (17) under the condition that the operators $T$ and $S$ are quasi-nonexpansive mappings.

Chang, Wang and Qin [14] proposed the following iterative algorithm for finding a solution of SEFPP (13):

$$
\begin{aligned}
u_{n} & =x_{n}-\gamma_{n} A^{*}\left(A x_{n}-B y_{n}\right) \\
x_{n+1} & =\alpha_{n} x_{n}+\left(1-\alpha_{n}\right)((1-\xi) I+\xi T((1-\eta) I+\eta T)) u_{n} \quad n \in \mathbb{N} . \\
v_{n} & =y_{n}+\beta_{n} B^{*}\left(A x_{n}-B y_{n}\right) \\
y_{n+1} & =\alpha_{n} y_{n}+\left(1-\alpha_{n}\right)((1-\xi) I+\xi S((1-\eta) I+\eta S)) v_{n}
\end{aligned}
$$

They established the weak convergence of the scheme (18) under the condition that the operators $T$ and $S$ are quasi-pseudocontractive mappings.

Boikanyo and Zegeye [6] proposed the following iterative algorithm for finding a solution of SEFPP (13):

$$
\begin{aligned}
u_{n} & =P_{C}\left[x_{n}-\gamma_{n} A^{*}\left(A x_{n}-B y_{n}\right)\right] \\
x_{n+1} & =\alpha_{n} u+\left(1-\alpha_{n}\right)((1-\xi) I+\xi T((1-\eta) I+\eta T)) u_{n} \quad \\
v_{n} & =P_{D}\left[y_{n}-\gamma_{n} B^{*}\left(A x_{n}-B y_{n}\right)\right] \\
y_{n+1} & =\alpha_{n} v+\left(1-\alpha_{n}\right)((1-\xi) I+\xi S((1-\eta) I+\eta S)) v_{n}
\end{aligned}
$$

They also established the strong convergence of the scheme (19) under the condition that the operators $T$ and $S$ are quasi-pseudocontractive mappings.

Motivated by the above works, we propose a new iterative algorithm called Halpern-type algorithm for the class of multivalue Lipschitzian quasi-pseudo-contractive mappings and maximal monotone operators that always converge strongly to the solution of the split equality fixed point and null point problem (SEFPNPP). It is known that the class of multivalue Lipschitzian quasi-pseudo-contractive mappings is more general than the class of quasi-contractive mappings, directed mappings, and demicontractive mappings. Moreover, strong convergence is more desirable than weak convergence and we obtain our result without additional conditions on the operators. Also, the implementation of the iterative algorithm does not require the calculation or estimation of the operator norms $\|A\|$ and $\|B\|$ which may at times be as difficult as solving the original problem itself. Hence, our results provide a unified framework for the study of the split equality fixed point and null point problem. 


\section{Preliminaries}

Let $H$ be a real Hilbert space with inner product $\langle\cdot, \cdot\rangle$ and norm $\|\cdot\|$, and let $C$ be a nonempty closed convex subset of $H$. The notation $x_{n} \rightarrow x$ denotes that the sequence $\left\{x_{n}\right\}$ converges strongly to $x$. Similarly, $x_{n} \rightarrow x$ will mean weak convergence.

For any $x \in H$, there exists a unique point $P_{C} x \in C$ such that

$$
\left\|x-P_{C} x\right\| \leq\|x-y\|, \forall y \in C .
$$

$P_{C}$ is called the metric projection of $H$ onto $C$. Note that $P_{C}$ is a nonexpansive mapping of $H$ onto $C$. For $x \in H$ and $z \in C$, we have

$$
z=P_{C} x \Leftrightarrow\langle z-y, x-z\rangle \geq 0 \text {, for every } y \in C .
$$

In [6], it was shown that if $H_{1}, H_{2}$ are real Hilbert spaces, then $H:=H_{1} \times H_{2}$ is also a real Hilbert space with inner product

$$
\left\langle\left(x_{1}, y_{1}\right),\left(x_{2}, y_{2}\right)\right\rangle=\left\langle x_{1}, x_{2}\right\rangle+\left\langle y_{1}, y_{2}\right\rangle, \quad \forall\left(x_{1}, y_{1}\right),\left(x_{2}, y_{2}\right) \in H_{1} \times H_{2}
$$

such that

$$
\left(x_{n}, y_{n}\right) \rightarrow\left(x^{*}, y^{*}\right) \text { implies that } x_{n} \rightarrow x^{*} \text { and } y_{n} \rightarrow y^{*}
$$

Moreover, if $C$ is a nonempty, closed, and convex subset of $H,(u, v) \in H$ and $\left(u^{*}, v^{*}\right)=$ $P_{C}(u, v)$, then from inequality $(21)$, we obtain that

$$
\left\langle\left(u^{*}, v^{*}\right)-(x, y),(u, v)-\left(u^{*}, v^{*}\right)\right\rangle \geq 0, \text { for every } y \in C, \quad \forall(x, y) \in H .
$$

Given a positive constant $\alpha$, a mapping $A: C \rightarrow H$ is said to be $\alpha$-inverse strongly monotone if

$$
\langle x-y, A x-A y\rangle \geq \alpha\|A x-A y\|^{2} \forall x, y \in C .
$$

For $\bar{\gamma}>0$, a mapping $A$ on $H$ is called $\bar{\gamma}$-strongly monotone if

$$
\langle x-y, A x-A y\rangle \geq \bar{\gamma}\|x-y\|^{2} \forall x, y \in H .
$$

Taking $L>0$, a mapping $A$ on $H$ is said to be $L$-Lipschitzian continuous if

$$
\|A x-A y\| \leq L\|x-y\|, \forall x, y \in H .
$$

It can be seen that $A$ is $\frac{\bar{\gamma}}{L^{2}}$-inverse strongly monotone whenever $A$ is $\bar{\gamma}$-strongly monotone and $L$-Lipschitzian continuous.

Let $B$ be a mapping of $H$ into $2^{H}$. The effective domain of $B$ is denoted by $\operatorname{dom}(B)$, that is, $\operatorname{dom}(B)=\{x \in H: B x \neq \emptyset\}$. A multivalued mapping $B$ is said to be monotone if

$$
\langle x-y, u-v\rangle \geq 0 \forall x, y \in \operatorname{dom}(B), u \in B x, v \in B y
$$

A monotone operator $B$ is said to be maximal if its graph is not properly contained in the graph of any other monotone operator. For a maximal monotone operator $B$ on $H$ and $r>0$, the operator

$$
J_{r}=(I+r B)^{-1}: H \rightarrow \operatorname{dom}(B)
$$

is called the resolvent of $B$ for $r$. It is known that $J_{r}$ is firmly nonexpansive. 
An operator $h$ is called averaged (see [3]) if there exists a nonexpansive operator $N: D \rightarrow H$ and a number $\alpha \in(0,1)$ such that

$$
h=(1-\alpha) I+\alpha N
$$

where $I$ is the identity operator.

Definition 2.1. Let $T: H \rightarrow H, I-T$ is called demiclosed at zero, if for any sequence $\left\{x_{n}\right\} \subset H$ and $x \in H$, we have $x_{n} \rightarrow x$ and $(I-T) x_{n} \rightarrow 0$, then $x \in F i x(T)$.

Lemma 2.1. [37] Let $H$ be a real Hilbert space, $C$ a closed convex subset of $H$. Let $T: C \rightarrow C$ be a continuous pseudocontractive mapping. Then

(i) $F(T)$ is a closed convex subset of $C$,

(ii) $(I-T)$ is demiclosed at zero.

Theorem 2.1. [21] Let $T: H \rightarrow H$ be a $\alpha$-attracting quasi-nonexpansive operator where $\alpha>0$ and $S: H \rightarrow H$ a strongly quasi-nonexpansive operator. Suppose that $F(T) \cap F(S) \neq \emptyset$. Then

(i) Both TS and ST are strongly quasi-nonexpansive,

(ii) If $I-T$ and $I-S$ are demiclosed at zero, then $I-T S$ and $I-S T$ are also demiclosed at zero.

Lemma 2.2. [15] Let $T: H \rightarrow H$ be a strictly quasi-nonexpansive operator and $S: H \rightarrow H$ a quasi-nonexpansive operator. Suppose that $F(T) \cap F(S) \neq \emptyset$. Then $F(T S)=F(S T)=$ $F(T) \cap F(S)$.

Lemma 2.3. [30]. Let $\left\{s_{n}\right\}$ be a sequence of nonnegative real numbers satisfying

$$
s_{n+1} \leq\left(1-\alpha_{n}\right) s_{n}+\alpha_{n} \beta_{n}+\gamma_{n}, n \geq 0
$$

where $\left\{\alpha_{n}\right\},\left\{\beta_{n}\right\}$ and $\left\{\gamma_{n}\right\}$ satisfy the following conditions:

(i) $\left\{\alpha_{n}\right\} \subset[0,1], \sum_{n=1}^{\infty} \alpha_{n}=\infty$,

(ii) $\limsup _{n \rightarrow \infty} \beta_{n} \leq 0$,

(iii) $\gamma_{n} \geq 0, \sum_{n=1}^{\infty} \gamma_{n}<\infty$.

Then $\lim _{n \rightarrow \infty} s_{n}=0$.

Lemma 2.4. Let $H$ be a Hilbert space with inner product $\langle\cdot\rangle$ and norm $\|$ respectively. Then $\forall x, y \in H$,

$$
\begin{gathered}
\text { (i) }\|t x+(1-t) y\|^{2}=t\|x\|^{2}+(1-t)\|y\|^{2}-t(1-t)\|x-y\|^{2}, \forall t \in[0,1] . \\
\text { (ii) }\|x-y\|^{2}=\|x\|^{2}-2\langle x, y\rangle+\|y\|^{2}
\end{gathered}
$$

\section{Main Results}

Theorem 3.1. Let $H_{1}$ and $H_{2}$ be real Hilbert spaces. Let $B_{1}$ and $B_{2}$ be maximal monotone operators of $H_{1}$ into $2^{H_{1}}$ and $H_{2}$ into $2^{H_{2}}$ and $J_{\lambda}^{B_{1}}$ and $J_{\lambda}^{B_{2}}$ be resolvents of $B_{1}$ and $B_{2}$, respectively for $\lambda>0$. Let $A: H_{1} \rightarrow H_{3}$ and $B: H_{2} \rightarrow H_{3}$ be two bounded linear operators, and $S: H_{1} \rightarrow C B\left(H_{1}\right)$ be Multivalued Lipschitzian quasi-pseudocontractive and 
$\mathrm{T}: \mathrm{H}_{2} \rightarrow \mathrm{CB}\left(\mathrm{H}_{2}\right)$ be Multivalued Lipschitzian quasi-pseudocontractive such that $(I-S)$ and $(I-T)$ are demiclosed at zero. If the solution set of SEFPNPP is nonempty (that is, $\left.\Gamma=\left\{x \in F(S) \cap B_{1}^{-1} 0, y \in F(T) \cap B_{2}^{-1} 0, A x_{n}=B y_{n}\right\} \neq \emptyset\right)$. Suppose that $x_{0}, x_{1} \in H_{1}$ and $y_{0}, y_{1} \in H_{2}$ are chosen arbitrarily. Let $\left\{\left(x_{n}, y_{n}\right)\right\}$ be the iterative sequence generated by

$$
\begin{aligned}
x_{n+1} & =\beta_{n} x_{0}+\left(1-\beta_{n}\right) u_{n} \\
u_{n} & =\alpha_{n} x_{n}+\left(1-\alpha_{n}\right) \eta_{n} \\
y_{n+1} & =\beta_{n} y_{0}+\left(1-\beta_{n}\right) v_{n} \\
v_{n} & =\alpha_{n} y_{n}+\left(1-\alpha_{n}\right) \mu_{n}
\end{aligned}
$$

where $\eta_{n} \in S J_{\lambda}^{B_{1}}\left(x_{n}-\gamma A^{*}\left(A x_{n}-B y_{n}\right)\right), \mu_{n} \in T J_{\lambda}^{B_{2}}\left(y_{n}+\gamma B^{*}\left(A x_{n}-B y_{n}\right)\right)$, the parameter $\gamma$ and the sequences $\left\{\alpha_{n}\right\},\left\{\beta_{n}\right\} \subset(0,1)$ satisfying the conditions: (i) $\gamma \in\left(0, \min \left(\frac{2}{\|A\|^{2}}, \frac{2}{\|B\|^{2}}\right)\right)$, (ii) $\sum_{n=1}^{\infty} \alpha_{n}<\infty$, (iii) $\lim _{n \rightarrow \infty} \beta_{n}=0$ and (iv) $\sum_{n=1}^{\infty} \beta_{n}=\infty$. Then,

(a) $\lim _{n \rightarrow \infty} \Phi_{n}(p, q)$ exists for each $p, q \in \Gamma$,

(b) $\lim _{n \rightarrow \infty}\left\|x_{n}-\eta_{n}\right\|=\lim _{n \rightarrow \infty}\left\|y_{n}-\mu_{n}\right\|=0$,

(c) $\left\{x_{n}\right\}_{n=1}^{\infty}$ converges strongly to $p, q \in \Gamma$.

Proof : We use Lemma 2.4 (see also [26]) and the fact that $S$ and $T$ are $L$-Lipschitzians.

$$
\|t x+(1-t) y\|^{2}=t\|x\|^{2}+(1-t)\|y\|^{2}-t(1-t)\|x-y\|^{2}
$$

which holds $\forall x, y \in H_{1}$. Let $(p, q) \in \Gamma$, then using (33) and (34), we have

$$
\begin{gathered}
\left\|x_{n+1}-p\right\|^{2}=\left\|\beta_{n} x_{0}+\left(1-\beta_{n}\right) u_{n}-p\right\|^{2} \\
=\left\|\beta_{n}\left(x_{0}-p\right)+\left(1-\beta_{n}\right)\left(u_{n}-p\right)\right\|^{2} \\
=\beta_{n}\left\|x_{0}-p\right\|^{2}+\left(1-\beta_{n}\right)\left\|u_{n}-p\right\|^{2}-\beta_{n}\left(1-\beta_{n}\left\|u_{n}-x_{0}\right\|^{2} .\right. \\
\left\|u_{n}-p\right\|^{2}=\left\|\alpha_{n} x_{n}+\left(1-\alpha_{n}\right)\left(\eta_{n}-p\right)\right\|^{2} \\
=\left\|\alpha_{n}\left(x_{n}-p\right)+\left(1-\alpha_{n}\right)\left(\eta_{n}-p\right)\right\|^{2} \\
=\alpha_{n}\left\|x_{n}-p\right\|^{2}+\left(1-\alpha_{n}\right)\left\|\eta_{n}-p\right\|^{2} \\
\quad-\alpha_{n}\left(1-\alpha_{n}\right)\left\|\eta_{n}-x_{n}\right\|^{2} .
\end{gathered}
$$

Substitute equation (36) into (35) to obtain

$$
\begin{aligned}
\| x_{n+1}- & p\left\|^{2}=\beta_{n}\right\| x_{0}-p \|^{2}+\left(1-\beta_{n}\right)\left\{\alpha_{n}\left\|x_{n}-p\right\|^{2}\right. \\
& +\left(1-\alpha_{n}\right)\left\|\eta_{n}-p\right\|^{2}-\alpha_{n}\left(1-\alpha_{n}\left\|\eta_{n}-x_{n}\right\|^{2}\right\} \\
& -\beta_{n}\left(1-\beta_{n}\left\|y_{n}-x_{0}\right\|^{2}\right. \\
= & \beta_{n}\left\|x_{0}-p\right\|^{2}+\alpha_{n}\left(1-\beta_{n}\right)\left\|x_{n}-p\right\|^{2} \\
& +\left(1-\alpha_{n}\right)\left(1-\beta_{n}\right)\left\|\eta_{n}-p\right\|^{2} \\
& -\alpha_{n}\left(1-\alpha_{n}\right)\left(1-\beta_{n}\right)\left\|\eta_{n}-x_{n}\right\|^{2} \\
& -\beta_{n}\left(1-\beta_{n}\left\|u_{n}-x_{0}\right\|^{2} .\right.
\end{aligned}
$$


Since $S$ is quasi pseudocontractive, we have

$$
\begin{aligned}
& H^{2}\left(S J_{\lambda}^{B_{1}}\left(x_{n}-\gamma A^{*}\left(A x_{n}-B y_{n}\right)\right), p\right) \leq\left\|J_{\lambda}^{B_{1}}\left(x_{n}-\gamma A^{*}\left(A x_{n}-B y_{n}\right)\right)-p\right\|^{2} \\
& +d^{2}\left(S J_{\lambda}^{B_{1}}\left(x_{n}-\gamma A^{*}\left(A x_{n}-B y_{n}\right)\right), J_{\lambda}^{B_{1}}\left(x_{n}-\gamma A^{*}\left(A x_{n}-B y_{n}\right)\right)\right) \\
& \left.\left.\leq \| x_{n}-p-\gamma A^{*}\left(A x_{n}-B y_{n}\right)\right)\right) \\
& +d^{2}\left(S J_{\lambda}^{B_{1}}\left(x_{n}-\gamma A^{*}\left(A x_{n}-B y_{n}\right)\right), J_{\lambda}^{B_{1}}\left(x_{n}-\gamma A^{*}\left(A x_{n}-B y_{n}\right)\right)\right) \\
& \leq\left\|x_{n}-p\right\|^{2}+\gamma^{2}\left\|A^{*}\left(A x_{n}-B y_{n}\right)\right\|^{2}-2 \gamma\left\langle x_{n}-p, A^{*}\left(A x_{n}-B y_{n}\right)\right\rangle \\
& +d^{2}\left(S J_{\lambda}^{B_{1}}\left(x_{n}-\gamma A^{*}\left(A x_{n}-B y_{n}\right)\right), J_{\lambda}^{B_{1}}\left(x_{n}-\gamma A^{*}\left(A x_{n}-B y_{n}\right)\right)\right) \\
& =\left\|x_{n}-p\right\|^{2}+\gamma^{2}\left\|A^{*}\left(A x_{n}-B y_{n}\right)\right\|^{2}-2 \gamma\left\langle A x_{n}-A p, A x_{n}-B y_{n}\right\rangle \\
& +d^{2}\left(S J_{\lambda}^{B_{1}}\left(x_{n}-\gamma A^{*}\left(A x_{n}-B y_{n}\right)\right), J_{\lambda}^{B_{1}}\left(x_{n}-\gamma A^{*}\left(A x_{n}-B y_{n}\right)\right)\right) \\
& \left\|A^{*}\left(A x_{n}-B y_{n}\right)\right\|^{2}=\left\langle A^{*}\left(A x_{n}-B y_{n}\right), A^{*}\left(A x_{n}-B y_{n}\right)\right\rangle \\
& =\left\langle A A^{*}\left(A x_{n}-B y_{n}\right), A x_{n}-B y_{n}\right\rangle \\
& =\|A\|^{2}\left\|A x_{n}-B y_{n}\right\|^{2} \\
& d^{2}\left(S J_{\lambda}^{B_{1}}\left(x_{n}-\gamma A^{*}\left(A x_{n}-B y_{n}\right)\right), J_{\lambda}^{B_{1}}\left(x_{n}-\gamma A^{*}\left(A x_{n}-B y_{n}\right)\right)\right) \\
& =\| \eta_{n}-J_{\lambda}^{B_{1}}\left(x_{n}-\gamma A^{*}\left(A x_{n}-B y_{n}\right) \|^{2}\right. \\
& \| \eta_{n}-J_{\lambda}^{B_{1}}\left(x_{n}-\gamma A^{*}\left(A x_{n}-B y_{n}\right)\|=\| \eta_{n}-p+p-J_{\lambda}^{B_{1}}\left(x_{n}-\gamma A^{*}\left(A x_{n}-B y_{n}\right) \|\right.\right. \\
& \leq\left\|\eta_{n}-p\right\|+\| J_{\lambda}^{B_{1}}\left(x_{n}-\gamma A^{*}\left(A x_{n}-B y_{n}\right)-p \|\right. \\
& \leq\left\|\eta_{n}-p\right\|+\| J_{\lambda}^{B_{1}}\left(x_{n}-\gamma A^{*}\left(A x_{n}-B y_{n}\right)-p \|\right. \\
& \leq\left\|\eta_{n}-p\right\|+\left\|x_{n}-p\right\|+\gamma\left\|A^{*}\left(A x_{n}-B y_{n}\right)\right\| \\
& \leq\left\|\eta_{n}-x_{0}\right\|+\left\|x_{n}-x_{0}\right\|+2\left\|x_{n}-p\right\|+\gamma\left\|A^{*}\left(A x_{n}-B y_{n}\right)\right\|
\end{aligned}
$$

Substitute equation (39) into (41), we have

$$
\begin{aligned}
\| \eta_{n} & -J_{\lambda}^{B_{1}}\left(x_{n}-\gamma A^{*}\left(A x_{n}-B y_{n}\right) \|\right. \\
& \leq\left\|\eta_{n}-x_{0}\right\|+\left\|x_{n}-x_{0}\right\|+2\left\|x_{n}-p\right\|+\gamma\|A\|\left\|A x_{n}-B y_{n}\right\|
\end{aligned}
$$

therefore,

$$
\begin{aligned}
\| \eta_{n}- & J_{\lambda}^{B_{1}}\left(x_{n}-\gamma A^{*}\left(A x_{n}-B y_{n}\right) \|^{2}\right. \\
\leq & (3+\gamma\|A\|)\left\|\eta_{n}-x_{0}\right\|^{2} \\
& +\left(\frac{5}{2}+\gamma\|A\|\right)\left\|x_{n}-x_{0}\right\|^{2} \\
& +\left(\frac{5}{2}+\gamma\|A\|\right)\left\|x_{n}-p\right\|^{2} \\
& +\left(3 \gamma\|A\|+\gamma^{2}\|A\|^{2}\right)\left\|A x_{n}-B y_{n}\right\|^{2}
\end{aligned}
$$


Substitute equations (42) and (43) into (38) to obtain

$$
\begin{aligned}
d^{2}\left(S J _ { \lambda } ^ { B _ { 1 } } \left(x_{n}-\right.\right. & \left.\left.\gamma A^{*}\left(A x_{n}-B y_{n}\right)\right), J_{\lambda}^{B_{1}}\left(x_{n}-\gamma A^{*}\left(A x_{n}-B y_{n}\right)\right)\right) \\
\leq & \left\|x_{n}-p\right\|^{2}+\gamma^{2}\|A\|^{2}\left\|A x_{n}-B y_{n}\right\|^{2} \\
& -2 \gamma\left\langle A x_{n}-A p, A x_{n}-B y_{n}\right\rangle+(3+\gamma\|A\|)\left\|\eta_{n}-x_{0}\right\|^{2} \\
& +\left(\frac{5}{2}+\gamma\|A\|\right)\left\|x_{n}-x_{0}\right\|^{2} \\
& +\left(\frac{5}{2}+\gamma\|A\|\right)\left\|x_{n}-p\right\|^{2} \\
& +\left(3 \gamma\|A\|+\gamma^{2}\|A\|^{2}\right)\left\|A x_{n}-B y_{n}\right\|^{2} \\
= & \left(\frac{7}{2}+2 \gamma\|A\|\right)\left\|x_{n}-p\right\|^{2} \\
& +(3+\gamma\|A\|)]\left\|\eta_{n}-x_{0}\right\|^{2} \\
& +\left(\frac{5}{2}+\gamma\|A\|\right)\left\|x_{n}-x_{0}\right\|^{2} \\
& +\left(3 \gamma\|A\|+\gamma^{2}\|A\|^{2}\right)\left\|A x_{n}-B y_{n}\right\|^{2} \\
& -2 \gamma\left\langle A x_{n}-A p, A x_{n}-B y_{n}\right\rangle
\end{aligned}
$$

Substitute equation (44) into (37) to obtain

$$
\begin{aligned}
\| x_{n+1}- & p\left\|^{2} \leq \beta_{n}\right\| x_{0}-p\left\|^{2}+\alpha_{n}\left(1-\beta_{n}\right)\right\| x_{n}-p \|^{2} \\
& +\left(1-\alpha_{n}\right)\left(1-\beta_{n}\right)\left\{\left(\frac{7}{2}+2 \gamma\|A\|\right)\left\|x_{n}-p\right\|^{2}\right. \\
& +(3+\gamma\|A\|)]\left\|\eta_{n}-x_{0}\right\|^{2} \\
& +\left(\frac{5}{2}+\gamma\|A\|\right)\left\|x_{n}-x_{0}\right\|^{2} \\
& +\left(3 \gamma\|A\|+\gamma^{2}\|A\|^{2}\right)\left\|A x_{n}-B y_{n}\right\|^{2} \\
& \left.-2 \gamma\left\langle A x_{n}-A p, A x_{n}-B y_{n}\right\rangle\right\} \\
& -\alpha_{n}\left(1-\alpha_{n}\right)\left(1-\beta_{n}\right)\left\|\eta_{n}-x_{n}\right\|^{2} \\
& -\beta_{n}\left(1-\beta_{n}\left\|u_{n}-x_{0}\right\|^{2}\right. \\
= & \beta_{n}\left\|x_{0}-p\right\|^{2}+\alpha_{n}\left(1-\beta_{n}\right)\left\|x_{n}-p\right\|^{2} \\
& +\left(1-\alpha_{n}\right)\left(1-\beta_{n}\right)\left(\frac{7}{2}+2 \gamma\|A\|\right)\left\|x_{n}-p\right\|^{2} \\
& +\left(1-\alpha_{n}\right)\left(1-\beta_{n}\right)(3+\gamma\|A\|)\left\|\eta_{n}-x_{0}\right\|^{2} \\
& +\left(1-\alpha_{n}\right)\left(1-\beta_{n}\right)\left(\frac{5}{2}+\gamma\|A\|\right)\left\|x_{n}-x_{0}\right\|^{2} \\
& +\left(1-\alpha_{n}\right)\left(1-\beta_{n}\right)\left(3 \gamma\|A\|+\gamma^{2}\|A\|^{2}\right)\left\|A x_{n}-B y_{n}\right\|^{2} \\
& -2\left(1-\alpha_{n}\right)\left(1-\beta_{n}\right) \gamma\left\langle A x_{n}-A p, A x_{n}-B y_{n}\right\rangle \\
& -\alpha_{n}\left(1-\alpha_{n}\right)\left(1-\beta_{n}\right)\left\|\eta_{n}-x_{n}\right\|^{2} \\
& -\beta_{n}\left(1-\beta_{n}\left\|u_{n}-x_{0}\right\|^{2} .\right.
\end{aligned}
$$

$$
\begin{aligned}
\| u_{n}- & x_{0}\left\|^{2}=\right\| \alpha_{n} x_{n}+\left(1-\alpha_{n}\right) \eta_{n}-x_{0} \|^{2} \\
= & \left\|\alpha_{n}\left(x_{n}-x_{0}\right)+\left(1-\alpha_{n}\right)\left(\eta_{n}-x_{0}\right)\right\|^{2} \\
\leq & \alpha_{n}\left\|x_{n}-x_{0}\right\|^{2}+\left(1-\alpha_{n}\right)\left\|\eta_{n}-x_{0}\right\|^{2} \\
& -\alpha_{n}\left(1-\alpha_{n}\right)\left\|\eta_{n}-x_{n}\right\|^{2} .
\end{aligned}
$$


Substitute equation (46) into (45) to obtain

$$
\begin{aligned}
& \left\|x_{n+1}-p\right\|^{2} \leq \beta_{n}\left\|x_{0}-p\right\|^{2}+\alpha_{n}\left(1-\beta_{n}\right)\left\|x_{n}-p\right\|^{2} \\
& +\left(1-\alpha_{n}\right)\left(1-\beta_{n}\right)\left(\frac{7}{2}+2 \gamma\|A\|\right)\left\|x_{n}-p\right\|^{2} \\
& +\left(1-\alpha_{n}\right)\left(1-\beta_{n}\right)(3+\gamma\|A\|)\left\|\eta_{n}-x_{0}\right\|^{2} \\
& +\left(1-\alpha_{n}\right)\left(1-\beta_{n}\right)\left(\frac{5}{2}+\gamma\|A\|\right)\left\|x_{n}-x_{0}\right\|^{2} \\
& +\left(1-\alpha_{n}\right)\left(1-\beta_{n}\right)\left(3 \gamma\|A\|+\gamma^{2}\|A\|^{2}\right)\left\|A x_{n}-B y_{n}\right\|^{2} \\
& -2\left(1-\alpha_{n}\right)\left(1-\beta_{n}\right) \gamma\left\langle A x_{n}-A p, A x_{n}-B y_{n}\right\rangle \\
& -\alpha_{n}\left(1-\alpha_{n}\right)\left(1-\beta_{n}\right)\left\|\eta_{n}-x_{n}\right\|^{2} \\
& -\beta_{n}\left(1-\beta_{n}\left\{\alpha_{n}\left\|x_{n}-x_{0}\right\|^{2}+\left(1-\alpha_{n}\right)\left\|\eta_{n}-x_{0}\right\|^{2}\right.\right. \\
& \left.-\alpha_{n}\left(1-\alpha_{n}\right)\left\|\eta_{n}-x_{n}\right\|^{2}\right\} \\
& =\beta_{n}\left\|x_{0}-p\right\|^{2}+\alpha_{n}\left(1-\beta_{n}\right)\left\|x_{n}-p\right\|^{2} \\
& +\left(1-\alpha_{n}\right)\left(1-\beta_{n}\right)\left(\frac{7}{2}+2 \gamma\|A\|\right)\left\|x_{n}-p\right\|^{2} \\
& +\left(1-\alpha_{n}\right)\left(1-\beta_{n}\right)(3+\gamma\|A\|)\left\|\eta_{n}-x_{0}\right\|^{2} \\
& +\left(1-\alpha_{n}\right)\left(1-\beta_{n}\right)\left(\frac{5}{2}+\gamma\|A\|\right)\left\|x_{n}-x_{0}\right\|^{2} \\
& +\left(1-\alpha_{n}\right)\left(1-\beta_{n}\right)\left(3 \gamma\|A\|+\gamma^{2}\|A\|^{2}\right)\left\|A x_{n}-B y_{n}\right\|^{2} \\
& -2\left(1-\alpha_{n}\right)\left(1-\beta_{n}\right) \gamma\left\langle A x_{n}-A p, A x_{n}-B y_{n}\right\rangle \\
& -\alpha_{n}\left(1-\alpha_{n}\right)\left(1-\beta_{n}\right)\left\|\eta_{n}-x_{n}\right\|^{2} \\
& -\beta_{n}\left(1-\beta_{n}\right) \alpha_{n}\left\|x_{n}-x_{0}\right\|^{2}-\beta_{n}\left(1-\beta_{n}\right)\left(1-\alpha_{n}\right)\left\|\eta_{n}-x_{0}\right\|^{2} \\
& +\alpha_{n} \beta_{n}\left(1-\beta_{n}\right)\left(1-\alpha_{n}\right)\left\|\eta_{n}-x_{n}\right\|^{2} \\
& =\left[\alpha_{n}\left(1-\beta_{n}\right)+\left(1-\alpha_{n}\right)\left(1-\beta_{n}\right)\left(\frac{7}{2}+2 \gamma\|A\|\right)\right]\left\|x_{n}-p\right\|^{2} \\
& +\left(1-\alpha_{n}\right)\left(1-\beta_{n}\right)\left(3 \gamma\|A\|+\gamma^{2}\|A\|^{2}\right)\left\|A x_{n}-B y_{n}\right\|^{2} \\
& -2\left(1-\alpha_{n}\right)\left(1-\beta_{n}\right) \gamma\left\langle A x_{n}-A p, A x_{n}-B y_{n}\right\rangle \\
& +\left[\alpha_{n} \beta_{n}\left(1-\beta_{n}\right)\left(1-\alpha_{n}\right)-\alpha_{n}\left(1-\beta_{n}\right)\left(1-\alpha_{n}\right)\right]\left\|\eta_{n}-x_{n}\right\|^{2} \\
& -\left(1-\beta_{n}\right)\left[\alpha_{n} \beta_{n}-\left(1-\alpha_{n}\right)\left(\frac{5}{2}+\gamma\|A\|\right)\left\|x_{n}-x_{0}\right\|^{2}\right. \\
& -\left(1-\beta_{n}\right)\left(1-\alpha_{n}\right)\left[\beta_{n}-(3+\gamma\|A\|)\left\|\eta_{n}-x_{0}\right\|^{2}\right. \\
& =\left[1-\beta_{n}+\left(1-\alpha_{n}\right)\left(1-\beta_{n}\right)\left(\frac{5}{2}+2 \gamma\|A\|\right)\right]\left\|x_{n}-p\right\|^{2} \\
& +\left(1-\alpha_{n}\right)\left(1-\beta_{n}\right)\left(3 \gamma\|A\|+\gamma^{2}\|A\|^{2}\right)\left\|A x_{n}-B y_{n}\right\|^{2} \\
& -2\left(1-\alpha_{n}\right)\left(1-\beta_{n}\right) \gamma\left\langle A x_{n}-A p, A x_{n}-B y_{n}\right\rangle \\
& -\alpha_{n}\left(1-\beta_{n}\right)^{2}\left(1-\alpha_{n}\right)\left\|\eta_{n}-x_{n}\right\|^{2} \\
& +\left(1-\beta_{n}\right)\left[\left(1-\alpha_{n}\right)\left(\frac{5}{2}+\gamma\|A\|\right)-\alpha_{n} \beta_{n}\right]\left\|x_{n}-x_{0}\right\|^{2} \\
& +\left(1-\beta_{n}\right)\left(1-\alpha_{n}\right)\left[(3+\gamma\|A\|)-\beta_{n}\right]\left\|\eta_{n}-x_{0}\right\|^{2} .
\end{aligned}
$$




$$
\begin{aligned}
\| y_{n+1}= & q\left\|^{2}=\right\| \beta_{n} y_{0}+\left(1-\beta_{n}\right) v_{n}-p \|^{2} \\
= & \left\|\beta_{n}\left(y_{0}-q\right)+\left(1-\beta_{n}\right)\left(v_{n}-q\right)\right\|^{2} \\
= & \beta_{n}\left\|y_{0}-p\right\|^{2}+\left(1-\beta_{n}\right)\left\|v_{n}-p\right\|^{2} \\
& -\beta_{n}\left(1-\beta_{n}\left\|v_{n}-y_{0}\right\|^{2} .\right. \\
\| v_{n}- & q\left\|^{2}=\right\| \alpha_{n} y_{n}+\left(1-\alpha_{n}\right)\left(\mu_{n}-q\right) \|^{2} \\
= & \left\|\alpha_{n}\left(y_{n}-q\right)+\left(1-\alpha_{n}\right)\left(\mu_{n}-q\right)\right\|^{2} \\
= & \alpha_{n}\left\|y_{n}-q\right\|^{2}+\left(1-\alpha_{n}\right)\left\|\mu_{n}-q\right\|^{2} \\
& -\alpha_{n}\left(1-\alpha_{n}\right)\left\|\mu_{n}-y_{n}\right\|^{2} .
\end{aligned}
$$

Substitute equation (49) into (48) to obtain

$$
\begin{aligned}
\| y_{n+1}- & q\left\|^{2}=\beta_{n}\right\| y_{0}-p \|^{2}+\left(1-\beta_{n}\right)\left\{\alpha_{n}\left\|y_{n}-q\right\|^{2}+\left(1-\alpha_{n}\right)\left\|\mu_{n}-q\right\|^{2}\right. \\
& \left.-\alpha_{n}\left(1-\alpha_{n}\right)\left\|\mu_{n}-y_{n}\right\|^{2}\right\}-\beta_{n}\left(1-\beta_{n}\left\|y_{n}-y_{0}\right\|^{2}\right. \\
= & \beta_{n}\left\|y_{0}-q\right\|^{2}+\alpha_{n}\left(1-\beta_{n}\right)\left\|y_{n}-q\right\|^{2} \\
& +\left(1-\alpha_{n}\right)\left(1-\beta_{n}\right)\left\|T J_{\lambda}^{B_{2}}\left(y_{n}+\gamma B^{*}\left(A x_{n}-B y_{n}\right)\right)-p\right\|^{2} \\
& -\alpha_{n}\left(1-\alpha_{n}\right)\left(1-\beta_{n}\right)\left\|T J_{\lambda}^{B_{2}}\left(y_{n}+\gamma B^{*}\left(A x_{n}-B y_{n}\right)\right)-y_{n}\right\|^{2} \\
& -\beta_{n}\left(1-\beta_{n}\left\|v_{n}-y_{0}\right\|^{2} .\right.
\end{aligned}
$$

Since $T$ is quasi pseudocontractive, we have

$$
\begin{aligned}
& H^{2}\left(T J_{\lambda}^{B_{2}}\left(y_{n}+\gamma B^{*}\left(A x_{n}-B y_{n}\right)\right), q\right) \leq\left\|J_{\lambda}^{B_{2}}\left(y_{n}+\gamma B^{*}\left(A x_{n}-B y_{n}\right)\right)-q\right\|^{2} \\
&+d^{2}\left(T J_{\lambda}^{B_{2}}\left(y_{n}+\gamma B^{*}\left(A x_{n}-B y_{n}\right)\right), J_{\lambda}^{B_{2}}\left(y_{n}+\gamma B^{*}\left(A x_{n}-B y_{n}\right)\right)\right) \\
& \leq\left.\| y_{n}-q+\gamma B^{*}\left(A x_{n}-B y_{n}\right)\right) \|^{2} \\
&+\left\|\mu_{n}-J_{\lambda}^{B_{2}}\left(y_{n}+\gamma B^{*}\left(A x_{n}-B y_{n}\right)\right)\right\|^{2} \\
& \leq\left\|y_{n}-q\right\|^{2}+\gamma^{2}\left\|B^{*}\left(A x_{n}-B y_{n}\right)\right\|^{2}+2 \gamma\left\langle y_{n}-q, B^{*}\left(A x_{n}-B y_{n}\right)\right\rangle \\
&+\left\|\mu_{n}-J_{\lambda}^{B_{2}}\left(y_{n}+\gamma B^{*}\left(A x_{n}-B y_{n}\right)\right)\right\|^{2} \\
&=\left\|y_{n}-q\right\|^{2}+\gamma^{2}\left\|B^{*}\left(A x_{n}-B y_{n}\right)\right\|^{2}+2 \gamma\left\langle B y_{n}-B q, A x_{n}-B y_{n}\right\rangle \\
&+\left\|\mu_{n}-J_{\lambda}^{B_{2}}\left(y_{n}+\gamma B^{*}\left(A x_{n}-B y_{n}\right)\right)\right\|^{2} \\
&\left\|B^{*}\left(A x_{n}-B y_{n}\right)\right\|^{2}=\left\langle B^{*}\left(A x_{n}-B y_{n}\right), B^{*}\left(A x_{n}-B y_{n}\right)\right\rangle=\left\langle B B^{*}\left(A x_{n}-B y_{n}\right), A x_{n}-B y_{n}\right\rangle \\
&=\|B\|^{2}\left\|A x_{n}-B y_{n}\right\|^{2} \\
& \\
&=\| J_{\lambda}^{B_{2}}\left(y_{n}+\gamma B^{*}\left(A x_{n}-B y_{n}\right) \|\right. \\
&=\| \mu_{n}-q+q-J_{\lambda}^{B_{1}}\left(y_{n}+\gamma B^{*}\left(A x_{n}-B y_{n}\right) \|\right. \\
& \leq\left\|\mu_{n}-q\right\|+\| J_{\lambda}^{B_{2}}\left(y_{n}+\gamma B^{*}\left(A x_{n}-B y_{n}\right)-q \|\right. \\
& \leq\left\|\mu_{n}-q\right\|+\| J_{\lambda}^{B_{1}}\left(y_{n}+\gamma B^{*}\left(A x_{n}-B y_{n}\right)-p \|\right. \\
& \leq \mu_{n}\|+2\| y_{n}-q\|+\gamma\| B^{*}\left(A x_{n}-B y_{n}\right) \|
\end{aligned}
$$


Substitute equation (52) into (53), we have

$$
\begin{aligned}
\| \mu_{n} & -J_{\lambda}^{B_{2}}\left(y_{n}+\gamma B^{*}\left(A x_{n}-B y_{n}\right) \|\right. \\
& \leq\left\|\mu_{n}-y_{0}\right\|+\left\|y_{n}-y_{0}\right\|+2\left\|y_{n}-q\right\|+\gamma\|B\|\left\|A x_{n}-B y_{n}\right\|
\end{aligned}
$$

therefore,

$$
\begin{aligned}
\| \mu_{n}- & J_{\lambda}^{B_{1}}\left(y_{n}+\gamma B^{*}\left(A x_{n}-B y_{n}\right) \|^{2}\right. \\
\leq & (3+\gamma\|B\|)\left\|\mu_{n}-y_{0}\right\|^{2}+\left(\frac{5}{2}+\gamma\|B\|\right)\left\|y_{n}-y_{0}\right\|^{2} \\
& +\left(\frac{5}{2}+\gamma\|B\|\right)\left\|y_{n}-q\right\|^{2}+\left(3 \gamma\|B\|+\gamma^{2}\|B\|^{2}\right)\left\|A x_{n}-B y_{n}\right\|^{2} .
\end{aligned}
$$

Substitute equations (52) and (55) into (51) to obtain

$$
\begin{aligned}
H^{2}\left(T J _ { \lambda } ^ { B _ { 2 } } \left(y_{n}+\right.\right. & \left.\gamma B^{*}\left(A x_{n}-B y_{n}\right), q\right) \leq\left\|y_{n}-q\right\|^{2}+\gamma^{2}\|B\|^{2}\left\|A x_{n}-B y_{n}\right\|^{2} \\
& +2 \gamma\left\langle B y_{n}-B q, A x_{n}-B y_{n}\right\rangle+(3+\gamma\|B\|)\|(3+\gamma\|B\|)\| \mu_{n}-y_{0} \|^{2} \\
& +\left(\frac{5}{2}+\gamma\|B\|\right)\left\|y_{n}-y_{0}\right\|^{2}+\left(\frac{5}{2}+\gamma\|B\|\right)\left\|y_{n}-q\right\|^{2} \\
& +\left(3 \gamma\|B\|+\gamma^{2}\|B\|^{2}\right)\left\|A x_{n}-B y_{n}\right\|^{2} \\
= & \left.\left(\frac{7}{2}+2 \gamma\|A\|\right)\left\|y_{n}-q\right\|^{2}+(3+\gamma\|A\|)\right]\left\|\mu_{n}-y_{0}\right\|^{2} \\
& +\left(\frac{5}{2}+\gamma\|A\|\right)\left\|y_{n}-y_{0}\right\|^{2}+\left(3 \gamma\|B\|+\gamma^{2}\|B\|^{2}\right)\left\|A x_{n}-B y_{n}\right\|^{2} \\
& +2 \gamma\left\langle A x_{n}-A p, A x_{n}-B y_{n}\right\rangle
\end{aligned}
$$

Substitute equation (56) into (50) to obtain

$$
\begin{aligned}
\| y_{n+1}- & p\left\|^{2} \leq \beta_{n}\right\| y_{0}-q\left\|^{2}+\alpha_{n}\left(1-\beta_{n}\right)\right\| y_{n}-q \|^{2} \\
& +\left(1-\alpha_{n}\right)\left(1-\beta_{n}\right)\left\{\left(\frac{7}{2}+2 \gamma\|A\|\right)\left\|y_{n}-q\right\|^{2}\right. \\
& +(3+\gamma\|A\|)]\left\|\mu_{n}-y_{0}\right\|^{2} \\
& +\left(\frac{5}{2}+\gamma\|A\|\right)\left\|y_{n}-y_{0}\right\|^{2} \\
& +\left(3 \gamma\|B\|+\gamma^{2}\|B\|^{2}\right)\left\|A x_{n}-B y_{n}\right\|^{2} \\
& \left.+2 \gamma\left\langle A x_{n}-A p, A x_{n}-B y_{n}\right\rangle\right\} \\
& -\alpha_{n}\left(1-\alpha_{n}\right)\left(1-\beta_{n}\right)\left\|\mu_{n}-y_{n}\right\|^{2} \\
& -\beta_{n}\left(1-\beta_{n}\left\|v_{n}-y_{0}\right\|^{2}\right. \\
= & \beta_{n}\left\|y_{0}-q\right\|^{2}+\alpha_{n}\left(1-\beta_{n}\right)\left\|y_{n}-q\right\|^{2} \\
& +\left(1-\alpha_{n}\right)\left(1-\beta_{n}\right)\left(\frac{7}{2}+2 \gamma\|A\|\right)\left\|y_{n}-q\right\|^{2} \\
& +\left(1-\alpha_{n}\right)\left(1-\beta_{n}\right)(3+\gamma\|A\|)\left\|\mu_{n}-y_{0}\right\|^{2} \\
& +\left(1-\alpha_{n}\right)\left(1-\beta_{n}\right)\left(\frac{5}{2}+\gamma\|A\|\right)\left\|y_{n}-y_{0}\right\|^{2} \\
& +\left(1-\alpha_{n}\right)\left(1-\beta_{n}\right)\left(3 \gamma\|B\|+\gamma^{2}\|B\|^{2}\right)\left\|A x_{n}-B y_{n}\right\|^{2} \\
& +2\left(1-\alpha_{n}\right)\left(1-\beta_{n}\right) \gamma\left\langle A x_{n}-A p, A x_{n}-B y_{n}\right\rangle \\
& -\alpha_{n}\left(1-\alpha_{n}\right)\left(1-\beta_{n}\right)\left\|\mu_{n}-y_{n}\right\|^{2} \\
& -\beta_{n}\left(1-\beta_{n}\left\|v_{n}-y_{0}\right\|^{2} .\right. \\
&
\end{aligned}
$$




$$
\begin{aligned}
\| v_{n} & -y_{0}\left\|^{2}=\right\| \alpha_{n} y_{n}+\left(1-\alpha_{n}\right) \mu_{n}-y_{0} \|^{2} \\
& =\left\|\alpha_{n}\left(y_{n}-y_{0}\right)+\left(1-\alpha_{n}\right)\left(\mu_{n}-y_{0}\right)\right\|^{2} \\
& \leq \alpha_{n}\left\|y_{n}-y_{0}\right\|^{2}+\left(1-\alpha_{n}\right)\left\|\mu_{n}-y_{0}\right\|^{2}-\alpha_{n}\left(1-\alpha_{n}\right)\left\|\mu_{n}-y_{n}\right\|^{2}
\end{aligned}
$$

Substitute equation (58) into (57) to obtain

$$
\begin{aligned}
& \left\|y_{n+1}-q\right\|^{2} \leq \beta_{n}\left\|y_{0}-q\right\|^{2}+\alpha_{n}\left(1-\beta_{n}\right)\left\|y_{n}-q\right\|^{2} \\
& +\left(1-\alpha_{n}\right)\left(1-\beta_{n}\right)\left(\frac{7}{2}+2 \gamma\|A\|\right)\left\|y_{n}-q\right\|^{2} \\
& +\left(1-\alpha_{n}\right)\left(1-\beta_{n}\right)(3+\gamma\|A\|)\left\|\mu_{n}-y_{0}\right\|^{2} \\
& +\left(1-\alpha_{n}\right)\left(1-\beta_{n}\right)\left(\frac{5}{2}+\gamma\|A\|\right)\left\|y_{n}-y_{0}\right\|^{2} \\
& +\left(1-\alpha_{n}\right)\left(1-\beta_{n}\right)\left(3 \gamma\|B\|+\gamma^{2}\|B\|^{2}\right)\left\|A x_{n}-B y_{n}\right\|^{2} \\
& +2\left(1-\alpha_{n}\right)\left(1-\beta_{n}\right) \gamma\left\langle A x_{n}-A p, A x_{n}-B y_{n}\right\rangle \\
& -\alpha_{n}\left(1-\alpha_{n}\right)\left(1-\beta_{n}\right)\left\|\mu_{n}-y_{n}\right\|^{2} \\
& -\beta_{n}\left(1-\beta_{n}\left\{\alpha_{n}\left\|y_{n}-y_{0}\right\|^{2}+\left(1-\alpha_{n}\right)\left\|\mu_{n}-y_{0}\right\|^{2}\right.\right. \\
& \left.-\alpha_{n}\left(1-\alpha_{n}\right)\left\|\mu_{n}-y_{n}\right\|^{2}\right\} \\
& =\beta_{n}\left\|y_{0}-q\right\|^{2}+\alpha_{n}\left(1-\beta_{n}\right)\left\|y_{n}-q\right\|^{2} \\
& +\left(1-\alpha_{n}\right)\left(1-\beta_{n}\right)\left(\frac{7}{2}+2 \gamma\|B\|\right)\left\|y_{n}-q\right\|^{2} \\
& +\left(1-\alpha_{n}\right)\left(1-\beta_{n}\right)(3+\gamma\|B\|)\left\|\mu_{n}-y_{0}\right\|^{2} \\
& +\left(1-\alpha_{n}\right)\left(1-\beta_{n}\right)\left(\frac{5}{2}+\gamma\|B\|\right)\left\|y_{n}-y_{0}\right\|^{2} \\
& +\left(1-\alpha_{n}\right)\left(1-\beta_{n}\right)\left(3 \gamma\|B\|+\gamma^{2}\|B\|^{2}\right)\left\|A x_{n}-B y_{n}\right\|^{2} \\
& +2\left(1-\alpha_{n}\right)\left(1-\beta_{n}\right) \gamma\left\langle B x_{n}-B q, A x_{n}-B y_{n}\right\rangle \\
& -\alpha_{n}\left(1-\alpha_{n}\right)\left(1-\beta_{n}\right)\left\|\mu_{n}-y_{n}\right\|^{2} \\
& -\beta_{n}\left(1-\beta_{n}\right) \alpha_{n}\left\|y_{n}-y_{0}\right\|^{2}-\beta_{n}\left(1-\beta_{n}\right)\left(1-\alpha_{n}\right)\left\|\mu_{n}-y_{0}\right\|^{2} \\
& +\alpha_{n} \beta_{n}\left(1-\beta_{n}\right)\left(1-\alpha_{n}\right)\left\|\mu_{n}-y_{n}\right\|^{2} \\
& =\left[\alpha_{n}\left(1-\beta_{n}\right)+\left(1-\alpha_{n}\right)\left(1-\beta_{n}\right)\left(\frac{7}{2}+2 \gamma\|A\|\right)\right]\left\|y_{n}-q\right\|^{2} \\
& +\left(1-\alpha_{n}\right)\left(1-\beta_{n}\right)\left(3 \gamma\|B\|+\gamma^{2}\|B\|^{2}\right)\left\|A x_{n}-B y_{n}\right\|^{2} \\
& +2\left(1-\alpha_{n}\right)\left(1-\beta_{n}\right) \gamma\left\langle B y_{n}-B q, A x_{n}-B y_{n}\right\rangle \\
& +\left[\alpha_{n} \beta_{n}\left(1-\beta_{n}\right)\left(1-\alpha_{n}\right)-\alpha_{n}\left(1-\beta_{n}\right)\left(1-\alpha_{n}\right)\right]\left\|\mu_{n}-y_{n}\right\|^{2} \\
& -\left(1-\beta_{n}\right)\left[\alpha_{n} \beta_{n}-\left(1-\alpha_{n}\right)\left(\frac{5}{2}+\gamma\|B\|\right)\left\|y_{n}-y_{0}\right\|^{2}\right. \\
& -\left(1-\beta_{n}\right)\left(1-\alpha_{n}\right)\left[\beta_{n}-(3+\gamma\|B\|)\left\|\mu_{n}-y_{0}\right\|^{2}\right. \\
& =\left[1-\beta_{n}+\left(1-\alpha_{n}\right)\left(1-\beta_{n}\right)\left(\frac{5}{2}+2 \gamma\|B\|\right)\right]\left\|y_{n}-q\right\|^{2} \\
& +\left(1-\alpha_{n}\right)\left(1-\beta_{n}\right)\left(3 \gamma\|B\|+\gamma^{2}\|B\|^{2}\right)\left\|A x_{n}-B y_{n}\right\|^{2} \\
& +2\left(1-\alpha_{n}\right)\left(1-\beta_{n}\right) \gamma\left\langle B y_{n}-B q, A x_{n}-B y_{n}\right\rangle \\
& -\alpha_{n}\left(1-\beta_{n}\right)^{2}\left(1-\alpha_{n}\right)\left\|\mu_{n}-y_{n}\right\|^{2} \\
& +\left(1-\beta_{n}\right)\left[\left(1-\alpha_{n}\right)\left(\frac{5}{2}+\gamma\|B\|\right)-\alpha_{n} \beta_{n}\right]\left\|y_{n}-y_{0}\right\|^{2} \\
& +\left(1-\beta_{n}\right)\left(1-\alpha_{n}\right)\left[(3+\gamma\|B\|)-\beta_{n}\right]\left\|\mu_{n}-y_{0}\right\|^{2} .
\end{aligned}
$$




$$
\begin{aligned}
& \left\|x_{n+1}-p\right\|^{2}+\left\|y_{n+1}-q\right\|^{2} \\
& \leq\left[1-\beta_{n}+\left(1-\alpha_{n}\right)\left(1-\beta_{n}\right)\left(\frac{5}{2}+2 \gamma\|A\|\right)\right]\left\|x_{n}-p\right\|^{2} \\
& +\left(1-\alpha_{n}\right)\left(1-\beta_{n}\right)\left(3 \gamma\|A\|+\gamma^{2}\|A\|^{2}\right)\left\|A x_{n}-B y_{n}\right\|^{2} \\
& -2\left(1-\alpha_{n}\right)\left(1-\beta_{n}\right) \gamma\left\langle A x_{n}-A p, A x_{n}-B y_{n}\right\rangle \\
& -\alpha_{n}\left(1-\beta_{n}\right)^{2}\left(1-\alpha_{n}\right)\left\|\eta_{n}-x_{n}\right\|^{2} \\
& +\left(1-\beta_{n}\right)\left[\left(1-\alpha_{n}\right)\left(\frac{5}{2}+\gamma\|A\|\right)-\alpha_{n} \beta_{n}\right]\left\|x_{n}-x_{0}\right\|^{2} \\
& +\left(1-\beta_{n}\right)\left(1-\alpha_{n}\right)\left[(3+\gamma\|A\|)-\beta_{n}\right]\left\|\eta_{n}-x_{0}\right\|^{2} \\
& +\left[1-\beta_{n}+\left(1-\alpha_{n}\right)\left(1-\beta_{n}\right)\left(\frac{5}{2}+2 \gamma\|B\|\right)\right]\left\|y_{n}-q\right\|^{2} \\
& +\left(1-\alpha_{n}\right)\left(1-\beta_{n}\right)\left(3 \gamma\|B\|+\gamma^{2}\|B\|^{2}\right)\left\|A x_{n}-B y_{n}\right\|^{2} \\
& +2\left(1-\alpha_{n}\right)\left(1-\beta_{n}\right) \gamma\left\langle B y_{n}-B q, A x_{n}-B y_{n}\right\rangle \\
& -\alpha_{n}\left(1-\beta_{n}\right)^{2}\left(1-\alpha_{n}\right)\left\|\mu_{n}-y_{n}\right\|^{2} \\
& +\left(1-\beta_{n}\right)\left[\left(1-\alpha_{n}\right)\left(\frac{5}{2}+\gamma\|B\|\right)-\alpha_{n} \beta_{n}\right]\left\|y_{n}-y_{0}\right\|^{2} \\
& +\left(1-\beta_{n}\right)\left(1-\alpha_{n}\right)\left[(3+\gamma\|B\|)-\beta_{n}\right]\left\|\mu_{n}-y_{0}\right\|^{2} \\
& =\left[1-\beta_{n}+\left(1-\alpha_{n}\right)\left(1-\beta_{n}\right)\right]\left[\left(\frac{5}{2}+2 \gamma\|A\|\right)\left\|x_{n}-p\right\|^{2}+\left(\frac{5}{2}+2 \gamma\|B\|\right)\left\|y_{n}-q\right\|^{2}\right] \\
& +\left(1-\alpha_{n}\right)\left(1-\beta_{n}\right)\left[\left(3 \gamma\|A\|+\gamma^{2}\|A\|^{2}\right)+\left(3 \gamma\|B\|+\gamma^{2}\|B\|^{2}\right)\right]\left\|A x_{n}-B y_{n}\right\|^{2} \\
& -2 \gamma\left(1-\alpha_{n}\right)\left(1-\beta_{n}\right)\left\|A x_{n}-B y_{n}\right\|^{2} \\
& -\alpha_{n}\left(1-\alpha_{n}\right)\left(1-\beta_{n}\right)^{2}\left(\left\|\eta_{n}-x_{n}\right\|^{2}+\left\|\mu_{n}-y_{n}\right\|^{2}\right) \\
& +\left(1-\beta_{n}\right)\left[\left(\left(1-\alpha_{n}\right)\left(\frac{5}{2}+\gamma\|A\|\right)-\alpha_{n} \beta_{n}\right)\left\|x_{n}-x_{0}\right\|^{2}\right. \\
& \left.\left.+\left(\left(1-\alpha_{n}\right)\left(\frac{5}{2}+\gamma\|B\|\right)-\alpha_{n} \beta_{n}\right)\left\|y_{n}-y_{0}\right\|^{2}\right)\right] \\
& +\left(1-\beta_{n}\right)\left(1-\alpha_{n}\right)\left[\left((3+\gamma\|A\|)-\beta_{n}\right)\left\|\eta_{n}-x_{0}\right\|^{2}\right. \\
& \left.+\left((3+\gamma\|B\|)-\beta_{n}\right)\left\|\mu_{n}-y_{0}\right\|^{2}\right] \\
& =\left[1-\beta_{n}+\left(1-\alpha_{n}\right)\left(1-\beta_{n}\right)\right]\left[\left(\frac{5}{2}+2 \gamma\|A\|\right)\left\|x_{n}-p\right\|^{2}+\left(\frac{5}{2}+2 \gamma\|B\|\right)\left\|y_{n}-q\right\|^{2}\right] \\
& -\left(1-\alpha_{n}\right)\left(1-\beta_{n}\right)\left[\left(3 \gamma\|A\|+\gamma^{2}\|A\|^{2}\right)+\left(3 \gamma\|B\|+\gamma^{2}\|B\|^{2}\right)+2 \gamma\right]\left\|A x_{n}-B y_{n}\right\|^{2} \\
& -\alpha_{n}\left(1-\alpha_{n}\right)\left(1-\beta_{n}\right)^{2}\left(\left\|\eta_{n}-x_{n}\right\|^{2}+\left\|\mu_{n}-y_{n}\right\|^{2}\right) \\
& +\left(1-\beta_{n}\right)\left[\left(\left(1-\alpha_{n}\right)\left(\frac{5}{2}+\gamma\|A\|\right)-\alpha_{n} \beta_{n}\right)\left\|x_{n}-x_{0}\right\|^{2}\right. \\
& \left.\left.+\left(\left(1-\alpha_{n}\right)\left(\frac{5}{2}+\gamma\|B\|\right)-\alpha_{n} \beta_{n}\right)\left\|y_{n}-y_{0}\right\|^{2}\right)\right] \\
& +\left(1-\beta_{n}\right)\left(1-\alpha_{n}\right)\left[\left((3+\gamma\|A\|)-\beta_{n}\right)\left\|\eta_{n}-x_{0}\right\|^{2}\right. \\
& \text { (60) } \left.\quad+\left((3+\gamma\|B\|)-\beta_{n}\right)\left\|\mu_{n}-y_{0}\right\|^{2}\right] \text {. }
\end{aligned}
$$




$$
\begin{aligned}
\Phi_{n+1}(p, q) \leq & {\left[1-\delta_{n}\right] \Phi_{n}(p, q)+\left(1-\beta_{n}\right) \sigma_{n} } \\
& -\left(1-\alpha_{n}\right)\left(1-\beta_{n}\right)\left[\left(3 \gamma\|A\|+\gamma^{2}\|A\|^{2}\right)\right. \\
& \left.+\left(3 \gamma\|B\|+\gamma^{2}\|B\|^{2}\right)+2 \gamma\right]\left\|A x_{n}-B y_{n}\right\|^{2} \\
& -\alpha_{n}\left(1-\alpha_{n}\right)\left(1-\beta_{n}\right)^{2}\left(\left\|\eta_{n}-x_{n}\right\|^{2}+\left\|\mu_{n}-y_{n}\right\|^{2}\right) \\
& -\beta_{n}\left(1-\beta_{n}\right)\left[\alpha_{n}+\left(1-\alpha_{n}\right)(1-\gamma) L^{2}\right]\left(\left\|x_{n}-x_{0}\right\|^{2}+\left\|y_{n}-y_{0}\right\|^{2}\right)
\end{aligned}
$$

where

$$
\delta_{n}=\beta_{n}-\left(1-\alpha_{n}\right)\left(1-\beta_{n}\right) .
$$

By condition (ii) $\sum_{n=1}^{\infty} \beta_{n}=\infty$ and then $\sum_{n=1}^{\infty} \delta_{n}=\infty$. Hence from Lemma 2.3 that following $\lim _{n \rightarrow \infty} \Phi_{n}(p, q)$ exists, implies

$$
\lim _{n \rightarrow \infty}\left\|x_{n}-p\right\| \text { and } \lim _{n \rightarrow \infty}\left\|y_{n}-q\right\|
$$

From equation (61)

$$
\begin{aligned}
(1- & \left.\alpha_{n}\right)\left(1-\beta_{n}\right)\left[\left(3 \gamma\|A\|+\gamma^{2}\|A\|^{2}\right)\right. \\
& \left.+\left(3 \gamma\|B\|+\gamma^{2}\|B\|^{2}\right)+2 \gamma\right]\left\|A x_{n}-B y_{n}\right\|^{2} \\
& -\alpha_{n}\left(1-\alpha_{n}\right)\left(1-\beta_{n}\right)^{2}\left(\left\|\eta_{n}-x_{n}\right\|^{2}+\left\|\mu_{n}-y_{n}\right\|^{2}\right) \\
\leq & \Phi_{n}(p, q)-\delta_{n} \Phi_{n}(p, q)+\left(1-\beta_{n}\right) \sigma_{n} \\
& -\Phi_{n+1}(p, q) \rightarrow 0(\text { as } n \rightarrow \infty) .
\end{aligned}
$$

This implies that

$$
\begin{array}{r}
\lim _{n \rightarrow \infty}\left\|A x_{n}-B y_{n}\right\|=0 \\
\lim _{n \rightarrow \infty}\left\|\eta_{n}-x_{n}\right\|=0 \\
\lim _{n \rightarrow \infty}\left\|\mu_{n}-y_{n}\right\|=0
\end{array}
$$

Also,

$$
\lim _{n \rightarrow \infty} \Phi_{n+1}\left(x_{n}, y_{n}\right)=0
$$

It follows from equations (33)

$$
\begin{aligned}
& \left\|x_{n+1}-x_{n}\right\|=\left\|\beta_{n} x_{0}+\left(1-\beta_{n}\right) y_{n}-x_{n}\right\| \\
= & \left\|\beta_{n}\left(x_{0}-x_{n}\right)+\left(1-\beta_{n}\right)\left(u_{n}-x_{n}\right)\right\| \\
= & \| \beta_{n}\left(x_{0}-x_{n}\right)+\left(1-\beta_{n}\right)\left(\alpha_{n} x_{n}+\left(1-\alpha_{n}\right)\left(\eta_{n}-x_{n}\right) \|\right. \\
= & \left\|\beta_{n}\left(x_{0}-x_{n}\right)+\left(1-\beta_{n}\right)\left(1-\alpha_{n}\right)\left(\eta_{n}-x_{n}\right)\right\| \\
\leq & \beta_{n}\left\|x_{0}-p\right\|+\beta_{n}\left\|x_{n}-p\right\| \\
& +\left(1-\beta_{n}\right)\left(1-\alpha_{n}\right)\left\|\eta_{n}-x_{n}\right\|
\end{aligned}
$$




$$
\begin{aligned}
&\left\|y_{n+1}-y_{n}\right\|=\left\|\beta_{n} y_{0}+\left(1-\beta_{n}\right) v_{n}-y_{n}\right\| \\
&=\left\|\beta_{n}\left(y_{0}-y_{n}\right)+\left(1-\beta_{n}\right)\left(u_{n}-y_{n}\right)\right\| \\
&= \| \beta_{n}\left(y_{0}-y_{n}\right)+\left(1-\beta_{n}\right)\left(\alpha_{n} y_{n}+\left(1-\alpha_{n}\right)\left(\mu_{n}-y_{n}\right) \|\right. \\
&=\left\|\beta_{n}\left(y_{0}-y_{n}\right)+\left(1-\beta_{n}\right)\left(1-\alpha_{n}\right)\left(\mu_{n}-y_{n}\right)\right\| \\
& \leq \quad \beta_{n}\left\|y_{0}-p\right\|+\beta_{n}\left\|y_{n}-q\right\| \\
& \quad+\left(1-\beta_{n}\right)\left(1-\alpha_{n}\right)\left\|\mu_{n}-y_{n}\right\| \\
& \quad \\
& \quad \Phi_{n+1}\left(x_{n}\right)=\left\|x_{n+1}-x_{n}\right\|+\left\|y_{n+1}-y_{n}\right\| \\
& \leq \quad \beta_{n}\left\|x_{0}-p\right\|+\beta_{n}\left\|x_{n}-p\right\| \\
& \quad+\left(1-\beta_{n}\right)\left(1-\alpha_{n}\right)\left\|\eta_{n}-x_{n}\right\| \\
& \quad+\beta_{n}\left\|y_{0}-q\right\|+\beta_{n}\left\|y_{n}-q\right\| \\
& \quad+\left(1-\beta_{n}\right)\left(1-\alpha_{n}\right)\left\|\mu_{n}-y_{n}\right\|
\end{aligned}
$$

From equations (62), (65) and (66), $\lim _{n \rightarrow \infty} \Phi_{n+1}\left(x_{n}, y_{n}\right)=0$.

By Lemma 2.1, we have $F(S), F\left(J_{\lambda}^{B_{1}}\right), F(T)$ and $F\left(J_{\lambda}^{B_{2}}\right)$ are closed and convex, and hence $\Gamma$ is also closed and convex. Let $(\hat{p}, \hat{q})=P_{\Gamma}(u, v)$. By characterization of the metric projection, we get

$$
\langle(u, v)-(\hat{p}, \hat{q}),(x, y)-(\hat{p}, \hat{q})\rangle \leq 0 ., \quad \forall z \in \Gamma .
$$

Now, since $\left\{x_{n}, y_{n}\right\}$ is bounded in $H_{1} \times H_{2}$, there exists $(\hat{p}, \hat{q}) \in H_{1} \times H_{2}$ and a subsequence $\left\{x_{n_{i}}, y_{n_{i}}\right\}$ of $\left\{x_{n}, y_{n}\right\}$ such that $\left(x_{n_{i}}, y_{n_{i}}\right) \rightarrow(\hat{p}, \hat{q})$ and Since $(\hat{p}, \hat{q}) \in \Gamma$, we obtain $S \hat{p}=\{\hat{p}\}$, $T \hat{q}=\{\hat{q}\}, J_{\lambda}^{B_{1}} \hat{p}=\{\hat{p}\}$ and $J_{\lambda}^{B_{2}} \hat{q}=\{\hat{q}\}$.

$$
\lim \sup _{n \rightarrow \infty}\left\langle(u, v)-(\hat{p}, \hat{q}),\left(x_{n}, y_{n}\right)-(\hat{p}, \hat{q})\right\rangle \leq 0 .
$$

To show this, since $\left\{x_{n}, y_{n}\right\}$ is bounded in $H_{1} \times H_{2}$, there exists $(\hat{p}, \hat{q}) \in H_{1} \times H_{2}$ and a subsequence $\left\{x_{n_{i}}, y_{n_{i}}\right\}$ of $\left\{x_{n}, y_{n}\right\}$ such that $\left(x_{n_{i}}, y_{n_{i}}\right) \rightarrow(\hat{p}, \hat{q})$ and

$$
\begin{aligned}
\lim \sup _{n \rightarrow \infty}[\langle u & \left.\left.-\hat{p}, x_{n}-\hat{p}\right\rangle+\left\langle v-\hat{q}, y_{n}-\hat{q}\right\rangle\right] \\
& =\lim _{n \rightarrow \infty}\left\langle(u, v)-(\hat{p}, \hat{q}),\left(x_{n}, y_{n}\right)-(\hat{p}, \hat{q})\right\rangle \\
& =\lim _{i \rightarrow \infty}\left\langle(u, v)-(\hat{p}, \hat{q}),\left(x_{n_{i}}, y_{n_{i}}\right)-(\hat{p}, \hat{q})\right\rangle .
\end{aligned}
$$

But $\left(x_{n_{i}}, y_{n_{i}}\right) \rightarrow(\hat{p}, \hat{q})$ implies that $x_{n_{i}} \rightarrow \hat{p}$ and $y_{n_{i}} \rightarrow \hat{q}$. Hence from equation (62), we have $x_{n_{i}} \rightarrow \hat{p}$ and $v_{n_{i}} \rightarrow \hat{q}$, respectively. Now, since $(I-S)$ and $(I-T)$ are demiclosed at zero, from Equation (65) and (66) we get $\hat{p} \in F(S)$ and $\hat{q} \in F(T)$.

Next, we show that $A \hat{p}=B \hat{a}$. Observe that

$$
\begin{aligned}
\| A \hat{p} & -B \hat{q}\left\|^{2}=\right\| A \hat{p}-A x_{n_{i}}+A x_{n_{i}}-B y_{n_{i}}+B y_{n_{i}}-B \hat{q} \|^{2} \\
& =\left\|\left(A \hat{p}-A x_{n_{i}}+B y_{n_{i}}-B \hat{q}\right)+\left(A x_{n_{i}}-B y_{n_{i}}\right)\right\|^{2} \\
& \leq\left\|A x_{n_{i}}-B y_{n_{i}}\right\|^{2}+2\left\langle A \hat{p}-B \hat{q}, A \hat{p}-A x_{n_{i}}+B y_{n_{i}}-B \hat{q}\right\rangle
\end{aligned}
$$


Since $x_{n_{i}} \rightarrow \hat{p}$ and $y_{n_{i}} \rightarrow \hat{q}$ as $i \rightarrow \infty$, it follows that $A x_{n_{i}} \rightarrow A \hat{p}$ and $B y_{n_{i}} \rightarrow B \hat{q}$ as $i \rightarrow \infty$ Taking limits on both sides, and making use of Equation (64), we get

$$
\begin{aligned}
\| A \hat{p}- & B \hat{q}\left\|^{2} \leq \limsup _{i \rightarrow \infty}\right\| A x_{n_{i}}-B y_{n_{i}} \|^{2} \\
& +2 \lim \sup _{i \rightarrow \infty}\left\langle A \hat{p}-B \hat{q}, A \hat{p}-A x_{n_{i}}+B y_{n_{i}}-B \hat{q}\right\rangle \\
= & 0 .
\end{aligned}
$$

The inequality (74) implies that $A \hat{p}=B \hat{q}$, that is $(x, y) \in \Gamma$.

Since $x_{n_{i}} \rightarrow \hat{p}, y_{n_{i}} \rightarrow \hat{q},\left\|\eta_{n}-x_{n}\right\| \rightarrow 0$ and $\left\|\mu_{n}-y_{n}\right\| \rightarrow 0$ as $n \rightarrow \infty$, we have $x_{n_{i}} \rightarrow \hat{p}$ and $y_{n_{i}} \rightarrow \hat{q}$. By the demiclosedness of $I-S$ and $I-J_{\lambda}^{B_{1}}$ at zero, then $I-S J_{\lambda}^{B_{2}}$ is also demiclosed at zero. Again by the demiclosedness of $I-T$ and $I-J_{\lambda}^{B_{2}}$ at zero, then $I-T J_{\lambda}^{B_{2}}$ is also demiclosed at zero, and from equations (65) and (66), we get $\hat{p} \in F\left(S J_{\lambda}^{B_{1}}\right)=F(S) \cap B_{1}^{-1} 0$ and $\hat{q} \in F\left(T J_{\lambda}^{B_{2}}\right)=F(T) \cap B_{2}^{-1} 0$.

Now let us show that $\hat{p} \in B_{1}^{-1} 0$. Let $\omega_{n}=J_{\lambda}^{B_{1}}\left(x_{n}-\gamma A^{*}\left(A x_{n}-B y_{n}\right)\right.$, then we can easily prove that

$$
\frac{1}{\lambda}\left(x_{n}-\omega_{n}-\gamma A^{*}\left(A x_{n}-B y_{n}\right)\right) \in B_{1} \omega_{n}
$$

By the monotonicity of $B_{1}$, we have

$$
\left\langle\omega_{n}-v, \frac{1}{\lambda}\left(x_{n}-\omega_{n}-\gamma A^{*}\left(A x_{n}-B y_{n}\right)\right)-w\right\rangle
$$

for all $(v, w) \in G\left(B_{1}\right)$. Thus, we also have

$$
\left\langle\omega_{n_{i}}-v, \frac{1}{\lambda}\left(x_{n_{i}}-\omega_{n_{i}}-\gamma A^{*}\left(A x_{n_{i}}-B y_{n_{i}}\right)\right)-w\right\rangle
$$

for all $(v, w) \in G\left(B_{1}\right)$. Since $\omega_{n_{i}} \rightarrow \hat{p},\left\|\omega_{n_{i}}-J_{\lambda}^{B B_{1}}\left(x_{n_{i}}-\gamma A^{*}\left(A x_{n_{i}}-B y_{n_{i}}\right)\right)\right\| \rightarrow 0$. A $x_{n_{i}}-B y_{n_{i}} \rightarrow$ 0 as $i \rightarrow \infty$, then by taking the limit as $i \rightarrow \infty$ in equation (75) yields

$$
\langle\hat{p}-v,-w\rangle \leq 0
$$

for all $(v, w) \in G\left(B_{1}\right)$. By the maximal monotonicity of $B_{1}$, we get $0 \in B_{1} \hat{p}$, that is, $\hat{p} \in B_{1}^{-1} 0$.

Again, let us show that $\hat{q} \in B_{2}^{-1} 0$. Let $\varpi_{n}=J_{\lambda}^{B_{2}}\left(y_{n}+\gamma B^{*}\left(A x_{n}-B y_{n}\right)\right.$, then we can easily prove that

$$
\frac{1}{\lambda}\left(y_{n}-\varpi_{n}+\gamma B^{*}\left(A x_{n}-B y_{n}\right)\right) \in B_{2} \varpi_{n}
$$

By the monotonicity of $B_{2}$, we have

$$
\left\langle\varpi_{n}-\varrho, \frac{1}{\lambda}\left(y_{n}-\varpi_{n}+\gamma B^{*}\left(A x_{n}-B y_{n}\right)\right)-\vartheta\right\rangle
$$

for all $(\varrho, \vartheta) \in G\left(B_{1}\right)$. Thus, we also have

$$
\left\langle\varpi_{n_{i}}-\varrho, \frac{1}{\lambda}\left(y_{n_{i}}-\varpi_{n_{i}}+\gamma B^{*}\left(A x_{n_{i}}-B y_{n_{i}}\right)\right)-\vartheta\right\rangle
$$

for all $(\varrho, \vartheta) \in G\left(B_{2}\right)$. Since $\varpi_{n_{i}} \rightarrow \hat{q},\left\|\varpi_{n_{i}}-J_{\lambda}^{B_{2}}\left(y_{n_{i}}-\gamma B^{*}\left(A x_{n_{i}}-B y_{n_{i}}\right)\right)\right\| \rightarrow 0 . A x_{n_{i}}-B y_{n_{i}} \rightarrow$ 0 as $i \rightarrow \infty$, then by taking the limit as $i \rightarrow \infty$ in equation (75) yields

$$
\langle\hat{q}-\varrho,-\vartheta\rangle \leq 0
$$


for all $(\varrho, \vartheta) \in G\left(B_{2}\right)$. By the maximal monotonicity of $B_{2}$, we get $0 \in B_{2} \hat{q}$, that is, $\hat{q} \in B_{2}^{-1} 0$. Hence, the sequence $\left\{\left(x_{n}, y_{n}\right)\right\}$ generated by equation (33) converges strongly to $(p . q) \in \Gamma$ as $n \rightarrow \infty$. This completes the proof of the theorem.

Corollary 3.1. Let $H_{1}$ and $H_{2}$ be real Hilbert spaces. Let $A: H_{1} \rightarrow H_{3}$ and $B: H_{2} \rightarrow$ $H_{3}$ be two bounded linear operators, and $S: H_{1} \rightarrow C B\left(H_{1}\right)$ be multivalued Lipschitzian quasipseudocontractive and $\mathrm{T}: \mathrm{H}_{2} \rightarrow \mathrm{CB}\left(\mathrm{H}_{2}\right)$ be multivalued Lipschitzian quasi-pseudocontractive such that $(I-S)$ and $(I-T)$ are demiclosed at zero. If the solution set of SEFPNPP is nonempty (that is, $\Gamma=\left\{x \in F(S), y \in F(T), A x_{n}=B y_{n}\right\} \neq \emptyset$ ). Suppose that $x_{0}, x_{1} \in H_{1}$ and $y_{0}, y_{1} \in H_{2}$ are chosen arbitrarily. Let $\left\{\left(x_{n}, y_{n}\right)\right\}$ be the iterative sequence generated by

$$
\begin{array}{rll}
x_{n+1} & =\beta_{n} x_{0}+\left(1-\beta_{n}\right) u_{n} & \\
u_{n} & =\alpha_{n} x_{n}+\left(1-\alpha_{n}\right) S\left(x_{n}-\gamma A^{*}\left(A x_{n}-B y_{n}\right)\right) & \\
y_{n+1} & =\beta_{n} y_{0}+\left(1-\beta_{n}\right) v_{n} & \\
v_{n} & =\alpha_{n} y_{n}+\left(1-\alpha_{n}\right) T\left(y_{n}+\gamma B^{*}\left(A x_{n}-B y_{n}\right)\right) &
\end{array}
$$

where the parameter $\gamma$ and the sequences $\left\{\alpha_{n}\right\},\left\{\beta_{n}\right\} \subset(0,1)$ satisfying the conditions: (i) $\gamma \in\left(0, \min \left(\frac{2}{\|A\|^{2}}, \frac{2}{\|B\|^{2}}\right)\right),($ ii $) \sum_{n=1}^{\infty} \alpha_{n}<\infty,($ iii $) \lim _{n \rightarrow \infty} \beta_{n}=0$ and $(i v) \sum_{n=1}^{\infty} \beta_{n}=\infty$. Then,

(a) $\lim _{n \rightarrow \infty} \Phi_{n}(p, q)$ exists for each $p, q \in \Gamma$,

(b) $\lim _{n \rightarrow \infty}\left\|x_{n}-S\left(x_{n}-\gamma A^{*}\left(A x_{n}-B y_{n}\right)\right)\right\|=\lim _{n \rightarrow \infty}\left\|y_{n}-T\left(y_{n}+\gamma B^{*}\left(A x_{n}-B y_{n}\right)\right)\right\|=0$,

(c) $\left\{x_{n}\right\}_{n=1}^{\infty}$ converges strongly to $(p, q) \in \Gamma$.

Corollary 3.2. Let $H_{1}$ and $H_{2}$ be real Hilbert spaces. Let $A: H_{1} \rightarrow H_{3}$ and $B: H_{2} \rightarrow H_{3}$ be two bounded linear operators and if the solution set of SEFPNPP is nonempty (that is, $\left.\Gamma=\left\{x \in B_{1}^{-1} 0, y \in B_{2}^{-1} 0, A x_{n}=B y_{n}\right\} \neq \emptyset\right)$. Suppose that $x_{0}, x_{1} \in H_{1}$ and $y_{0}, y_{1} \in H_{2}$ are chosen arbitrarily. Let $\left\{\left(x_{n}, y_{n}\right)\right\}$ be the iterative sequence generated by

$$
\begin{array}{rll}
x_{n+1} & =\beta_{n} x_{0}+\left(1-\beta_{n}\right) u_{n} & \\
u_{n} & =\alpha_{n} x_{n}+\left(1-\alpha_{n}\right) J_{\lambda}^{B_{1}}\left(x_{n}-\gamma A^{*}\left(A x_{n}-B y_{n}\right)\right) & \\
y_{n+1} & =\beta_{n} y_{0}+\left(1-\beta_{n}\right) v_{n} & \\
v_{n} & =\alpha_{n} y_{n}+\left(1-\alpha_{n}\right) J_{\lambda}^{B_{2}}\left(y_{n}+\gamma B^{*}\left(A x_{n}-B y_{n}\right)\right) &
\end{array}
$$

where the parameter $\gamma$ and the sequences $\left\{\alpha_{n}\right\},\left\{\beta_{n}\right\} \subset(0,1)$ satisfying the conditions: (i) $\gamma \in\left(0, \min \left(\frac{2}{\|A\|^{2}}, \frac{2}{\|B\|^{2}}\right)\right)$, (ii) $\sum_{n=1}^{\infty} \alpha_{n}<\infty$, (iii) $\lim _{n \rightarrow \infty} \beta_{n}=0$ and $($ iv $) \sum_{n=1}^{\infty} \beta_{n}=\infty$. Then,

(a) $\lim _{n \rightarrow \infty} \Phi_{n}(p, q)$ exists for each $p, q \in \Gamma$,

(b) $\lim _{n \rightarrow \infty}\left\|x_{n}-J_{\lambda}^{B_{1}}\left(x_{n}-\gamma A^{*}\left(A x_{n}-B y_{n}\right)\right)\right\|=\lim _{n \rightarrow \infty}\left\|y_{n}-J_{\lambda}^{B_{2}}\left(y_{n}+\gamma B^{*}\left(A x_{n}-B y_{n}\right)\right)\right\|=0$,

(c) $\left\{x_{n}\right\}_{n=1}^{\infty}$ converges strongly to $(p, q) \in \Gamma$.

\section{Applications}

Let $f$ be a bifunction from $C \times C$ to $\mathbb{R}$, where $\mathbb{R}$ is the set of real numbers. The equilibrium problem is to find $\bar{x} \in C$ such that $f(\bar{x}, y) \geq 0$ for all $y \in C$. The set of such solutions is denoted by $E P(f)$. Numerous problems in physics, optimization, and economics reduce to finding a solution to the equilibrium problem (see [5]).

Lemma 4.1. For solving the equilibrium problem, they assumed that the bifunction $f$ satisfies the following conditions:

(A1) $f(x, x)=0$ for all $x \in C$, 
(A2) $f$ is monotone, that is, $f(x, y)+f(y, x) \leq 0$ for all $x, y \in C$,

(A3) for every $x, y, z \in C$, $\lim _{\sup _{t \downarrow 0}} f(t z+(1-t) x, y) \leq f(x, y)$,

(A4) $f(x, \cdot)$ is convex and lower semicontinuous for each $x \in C$.

Lemma 4.2. [5]. Let $C$ be a nonempty closed convex subset of $H$, and let $f$ be a bifunction from $C \times C$ to $\mathbb{R}$ satisfying (A1) - (A4). If $r>0$ and $x \in H$, then there exists $z \in C$ such that

$$
f(z, y)+\frac{1}{r}\langle y-z, z-x\rangle \geq 0, \forall y \in C .
$$

Lemma 4.3. [19]. Let $C$ be a nonempty closed convex subset of $H$, and let $f$ be a bifunction from $C \times C$ to $\mathbb{R}$ satisfying (A1) - (A4). For $r>0$, define a mapping $T_{r}: H \rightarrow C$ as follows:

$$
T_{r}(x)=\left\{z \in C: f(z, y)+\frac{1}{r}\langle y-z, z-x\rangle \geq 0, \forall y \in C\right\} .
$$

Then the following hold:

(i) $T_{r}$ is single valued,

(ii) $T_{r}$ is firmly nonexpansive, that is, for any $x, y \in H$

$$
\left\langle x-y, T_{r} x-T_{r} y\right\rangle \geq\left\|T_{r} x-T_{r} y\right\|^{2},
$$

(iii) $\operatorname{Fix}\left(T_{r}\right)=E P(f)$,

(iv) $E P(f)$ is closed and convex.

Let $C$ and $Q$ be nonempty closed convex subsets of $H_{1}$ and $H_{2}$, respectively. Let $f_{1}: C \times C \rightarrow$ $\mathbb{R}$ and $f_{2}: Q \times Q \rightarrow \mathbb{R}$ be two bifunctions and $A: H_{1} \rightarrow H_{3}$ and $B: H_{2} \rightarrow H_{3}$ be bounded linear operators, then the split equality equilibrium problem (SEEP) is to find a point $\left(x^{*}, y^{*}\right) \in C \times Q$ such that

$$
\left.\begin{array}{l}
f_{1}\left(x^{*}, x\right) \geq 0 \forall x \in C \text { and } \\
f_{2}\left(y^{*}, y\right) \geq 0 \forall y \in Q
\end{array}\right\}
$$

Then above problem is to find a point $\left(x^{*}, y^{*}\right) \in C \times Q$ such that

$$
x^{*} \in E P\left(f_{1}\right) \text { and } y^{*} \in E P\left(f_{2}\right): A x^{*}=B y^{*} .
$$

Lemma 4.4. [29] Let $C$ be a nonempty closed convex subset of $H$, and let $f$ be a bifunction from $C \times C$ to $\mathbb{R}$ satisfying (A1) - (A4). Define $A_{f}$ as follows:

$$
A_{f}(x)=\left\{\begin{array}{lr}
\{z \in H: f(z, y) \geq\langle y-x, z\rangle, & \forall y \in C\}, \\
\emptyset & \text { if } x \in C \\
\text { if } \notin C
\end{array}\right.
$$

Then the following hold:

(i) $A_{f}$ is msximal monotone,

(ii) $\operatorname{EP}(f)=A_{f}^{-1} 0$,

(ii) $T_{r}^{f}=\left(I+r A_{f}\right)^{-1} 0, \quad r>0$.

Consider the variational inequality problem. Let $H$ be a real Hilbert space, and let $f$ be a proper lower semicontinuous convex function of $H$ into $(-\infty,+\infty]$. Then the subdifferential $\partial f$ of $f$ is defined as

$$
\partial f(x)=\{z \in H: f(y)-f(x) \geq\langle z, y-x\rangle, \forall y \in H\}
$$


for all $x \in H$. [27] claimed that $\partial f$ is a maximal monotone operator. Let $C$ be a nonempty closed convex subset of $H$, and let $\delta_{C}$ be the indicator function of $C$. That is,

$$
\delta_{C}(x)=\left\{\begin{array}{cc}
0 & x \in C \\
+\infty & x \notin C
\end{array}\right.
$$

Since $\delta_{C}$ is a proper lower semicontinuous convex function on $H$, the subdifferential $\partial_{\delta_{C}}$ of $\delta_{C}$ is a maximal monotone operator. The resolvent $J_{\lambda}$ of $\partial_{\delta_{C}}$ for $\lambda>0$ is defined by

$$
J_{\lambda} x=\left(I+\lambda \partial_{\delta_{C}}\right)^{-1} x, \quad \forall x \in H .
$$

they have

$$
\begin{aligned}
u=\left(I+\lambda \partial_{\delta_{C}}\right)^{-1} x & \Leftrightarrow x \in u+\lambda \partial_{\delta_{C}} u \\
& \Leftrightarrow x \in u+\lambda N_{C} u \Leftrightarrow x-u \in \lambda N_{C} u \\
& \Leftrightarrow \frac{1}{\lambda}\langle x-u, y-u\rangle \leq 0, \quad \forall y \in C \\
& \Leftrightarrow u=P_{C} x
\end{aligned}
$$

where $N_{C} u=\{z \in H:\langle z, x-u\rangle \leq 0 \forall y \in C\}$. The variational inequality problem for nonlinear operator $A$ is to find $z \in C$ such that

$$
\langle A z, y-z\rangle \geq 0 \forall y \in C
$$

The set of its solutions is denoted by $\operatorname{VI}(C, A)$. Then they have

$$
\begin{aligned}
z & \in V I(C, A) \\
& \Leftrightarrow\langle A z, x-z\rangle \geq 0 \forall y \in C \\
& \Leftrightarrow\langle-A z, x-z\rangle \leq 0 \quad \forall y \in C \\
& \Leftrightarrow-A z \in N_{C} z \\
& \Leftrightarrow 0 \in A z+N_{C} z \Leftrightarrow 0 \in A z+\partial_{\delta_{C}} z \\
& \Leftrightarrow z \in\left(A+\partial_{\delta_{C}}\right)^{-1} 0 .
\end{aligned}
$$

With (90), we can obtain the strong convergence theorem for the variational inequality problem.

Let $C$ and $Q$ be nonempty closed convex subsets of $H_{1}$ and $H_{2}$, respectively. Let $S: H_{1} \rightarrow H_{1}$ and $T: H_{2} \rightarrow H_{2}$ be two quasi pseudocontractiv mappings and $A: H_{1} \rightarrow H_{3}$ and $B: H_{2} \rightarrow H_{3}$ be bounded linear operators.

The Split Equality Variational Inequality Problem denoted by SEVIP is to find a point $\left(u^{*}, v^{*}\right) \in C \times Q$ such that

$$
\left.\begin{array}{l}
\left\langle u-u^{*}, S J_{\lambda}^{B_{1}}\left(u^{*}-\gamma A^{*}\left(A x_{n}-B y_{n}\right)\right)\right\rangle \geq 0 \forall u \in C \text { and } \\
\left\langle v-v^{*}, T J_{\lambda}^{B_{1}}\left(v^{*}+\gamma B^{*}\left(A x_{n}-B y_{n}\right)\right)\right\rangle \geq 0 \forall v \in Q . \\
\text { such that } A x_{n}=B y_{n} .
\end{array}\right\}
$$

Let $D$ be the solution set of the SEVIP given by

$$
D=\left\{u^{*} \in V I(C, S), v^{*} \in V I(Q, T): A x_{n}=B y_{n}\right\}
$$

We observe that $u^{*}, v^{*} \in S E V I P$ if and only if $u^{*}=S J_{\lambda}^{B_{1}}\left(u^{*}-\gamma A^{*}\left(A x_{n}-B y_{n}\right)\right)$ and $v^{*}=$ $S J_{\lambda}^{B_{1}}\left(v^{*}+\gamma B^{*}\left(A x_{n}-B y_{n}\right)\right)$. 
Let $f: H \rightarrow(-\infty,+\infty]$ be a function, we define the set of minimizer of $f$ by

$$
\operatorname{Argmin} f:=\{x \in H: f(x) \leq f(z), \forall z \in H\} .
$$

If $f$ is a proper, lower semicontinuous and convex function, then $\partial f$ is a maximal monotone operator. Moreover,

$$
x \in(\partial f)^{-1} 0 \Longleftrightarrow 0 \in \partial f(x) \Leftrightarrow f(x) \leq f(z), \forall z \in H \Leftrightarrow x \in \operatorname{Argmin} f,
$$

that is, Argmin $f=(\partial f)^{-1} 0$. In this case, the resolvent of $\partial f$ is called the proximity operator of $f$.

Let $H_{1}$ and $H_{2}$ be real Hilbert spaces. Let $f: H_{1} \rightarrow(-\infty,+\infty]$ and $g: H_{2} \rightarrow(-\infty,+\infty]$ be proper, lower semicontinuous and convex functions. Let $A: H_{1} \rightarrow H_{3}$ and $B: H_{2} \rightarrow H_{3}$ be bounded linear operators, the Split Optimizatin problem (SOP) is the problem of finding $\left(x^{*}, y^{*}\right) \in H_{1} \times H_{2}$ such that

$$
x^{*} \in \operatorname{Argmin} f \text { and } y^{*} \in \operatorname{Argmin} g \text {, such that } A x^{*}=B y^{*} .
$$

Denote by $\partial f=B_{1}$ and $\partial g=B_{2}$. Since $x^{*}$ and $y^{*}$ are the minimum of $f$ on $H_{1}$ and $g$ on $H_{2}$, respectively for any $\lambda>0$, we have

$$
\begin{array}{r}
x^{*}=F(S) \cap(\partial f)^{-1} 0=F i x\left(S J_{\lambda}^{\partial f}\right) \text { and } \\
y^{*}=F(T) \cap(\partial g)^{-1} 0=F i x\left(T J_{\lambda}^{\partial g}\right) .
\end{array}
$$

This implies that the split optimization problem (95) is equivalent to the split common fixed point and null point problem SEFPNPP.

\subsection{Split Equality feasibility Problem (SFP).}

Theorem 4.1. Let $H_{1}$ and $H_{2}$ be real Hilbert spaces and $C$ and $Q$ be nonempty closed convex subsets of $H_{1}$ and $H_{2}$ respectively. Let $A: H_{1} \rightarrow H_{3}$ and $B: H_{2} \rightarrow H_{3}$ be bounded linear operators, and $S: H_{1} \rightarrow C B\left(H_{1}\right)$ be Lipschitzian quasi-pseudocontractive and $T: H_{2} \rightarrow$ $C B\left(H_{2}\right)$ be Lipschitzian quasi-pseudocontractive such that $(I-S)$ and $(I-T)$ are demiclosed at zero. If the solution set of SFP is nonempty (that is, $\Gamma=\{x \in F(S) \cap C, y \in F(T) \cap Q$ : $\left.\left.A x_{n}=B y_{n}\right\} \neq \emptyset\right)$. Suppose that $x_{0}, x_{1} \in H_{1}$ and $y_{0}, y_{1} \in H_{2}$ are chosen arbitrarily. Let $\left\{\left(x_{n}, y_{n}\right)\right\}$ be the iterative sequence generated by

$$
\begin{array}{rll}
x_{n+1} & =\beta_{n} x_{0}+\left(1-\beta_{n}\right) u_{n} & \\
u_{n} & =\alpha_{n} x_{n}+\left(1-\alpha_{n}\right) S P_{C}\left(x_{n}-\gamma A^{*}\left(A x_{n}-B y_{n}\right)\right) & \\
y_{n+1} & =\beta_{n} y_{0}+\left(1-\beta_{n}\right) v_{n} & \\
v_{n} & =\alpha_{n} y_{n}+\left(1-\alpha_{n}\right) T P_{Q}\left(y_{n}+\gamma B^{*}\left(A x_{n}-B y_{n}\right)\right) &
\end{array}
$$

where the parameter $\gamma$ and the sequences $\left\{\alpha_{n}\right\},\left\{\beta_{n}\right\} \subset(0,1)$ satisfying the conditions: (i) $\gamma \in\left(0, \min \left(\frac{2}{\|A\|^{2}}, \frac{2}{\|B\|^{2}}\right)\right),($ ii $) \sum_{n=1}^{\infty} \alpha_{n}<\infty$, (iii) $\lim _{n \rightarrow \infty} \beta_{n}=0$ and $($ iv $) \sum_{n=1}^{\infty} \beta_{n}=\infty$. Then,

(a) $\lim _{n \rightarrow \infty} \Phi_{n}(p, q)$ exists for each $p, q \in \Gamma$,

(b) $\lim _{n \rightarrow \infty}\left\|x_{n}-S P_{C}\left(x_{n}-\gamma A^{*}\left(A x_{n}-B y_{n}\right)\right)\right\|=\lim _{n \rightarrow \infty}\left\|y_{n}-T P_{Q}\left(y_{n}+\gamma B^{*}\left(A x_{n}-B y_{n}\right)\right)\right\|=0$,

(c) $\left\{x_{n}\right\}_{n=1}^{\infty}$ converges strongly to $(p, q) \in \Gamma$.

Proof: Set $B_{1}:=\partial \delta_{C}$ and $B_{2}:=\partial \delta_{Q}$. Then $B_{1}$ and $B_{2}$ are maximal monotone such that $J_{\lambda}^{B_{1}}=P_{C}$ and $J_{\lambda}^{B_{2}}=P_{Q}$ for $\lambda>0$. We also have $B_{1}^{-1} 0=C$ and $B_{2}^{-1} 0=Q$. Hence the result is obtained directly by Theorem 3.1 . 


\subsection{Split Equality Equilibrium Problem (SEEP).}

Theorem 4.2. Let $C$ and $Q$ be nonempty closed convex subsets of $H_{1}$ and $H_{2}$, respectively. $f_{1}: C \times C \rightarrow \mathbb{R}$ and $f_{2}: C \times C \rightarrow \mathbb{R}$ be bifunctions satisfying (A1) - (A4) and let $T_{r_{1}}^{f_{1}}$ and $T_{r_{2}}^{f_{2}}$ be resolvents of $A_{f_{1}}$ and $A_{f_{2}}$ in Lemma 4.4, respectively for $r_{1}, r_{2}>0$. Let $A: H_{1} \rightarrow H_{3}$ and $B$ : $\mathrm{H}_{2} \rightarrow \mathrm{H}_{3}$ be bounded linear operators, and $S: H_{1} \rightarrow C B\left(H_{1}\right)$ be multivalued Lipschitzian quasipseudocontractive and $\mathrm{T}: \mathrm{H}_{2} \rightarrow \mathrm{CB}\left(\mathrm{H}_{2}\right)$ be multivalued Lipschitzian quasi-pseudocontractive such that $(I-S)$ and $(I-T)$ are demiclosed at zero. If the solution set of SEEP (83) is nonempty (that is, $\Gamma=\left\{x \in F(S) \cap E P\left(f_{1}\right), y \in F(T) \cap E P\left(f_{2}\right),: A x_{n}=B y_{n}\right\} \neq \emptyset$ ). Suppose that $x_{0}, x_{1} \in H_{1}$ and $y_{0}, y_{1} \in H_{2}$ are chosen arbitrarily. Let $\left\{\left(x_{n}, y_{n}\right)\right\}$ be the iterative sequence generated by

$$
\begin{array}{rll}
x_{n+1} & =\beta_{n} x_{0}+\left(1-\beta_{n}\right) u_{n} & \\
u_{n} & =\alpha_{n} x_{n}+\left(1-\alpha_{n}\right) S T_{r_{1}}^{f_{1}}\left(x_{n}-\gamma A^{*}\left(A x_{n}-B y_{n}\right)\right) & \\
y_{n+1} & =\beta_{n} y_{0}+\left(1-\beta_{n}\right) v_{n} & \\
v_{n} & =\alpha_{n} y_{n}+\left(1-\alpha_{n}\right) T T_{r_{2}}^{f_{2}}\left(y_{n}+\gamma B^{*}\left(A x_{n}-B y_{n}\right)\right) &
\end{array}
$$

where the parameter $\gamma$ and the sequences $\left\{\alpha_{n}\right\},\left\{\beta_{n}\right\} \subset(0,1)$ satisfying the conditions: (i) $\gamma \in\left(0, \min \left(\frac{2}{\|A\|^{2}}, \frac{2}{\|B\|^{2}}\right)\right),($ ii $) \sum_{n=1}^{\infty} \alpha_{n}<\infty$, (iii) $\lim _{n \rightarrow \infty} \beta_{n}=0$ and $(i v) \sum_{n=1}^{\infty} \beta_{n}=\infty$. Then,

(a) $\lim _{n \rightarrow \infty} \Phi_{n}(p, q)$ exists for each $p, q \in \Gamma$,

(b) $\lim _{n \rightarrow \infty}\left\|x_{n}-S T_{r_{1}}^{f_{1}}\left(x_{n}-\gamma A^{*}\left(A x_{n}-B y_{n}\right)\right)\right\|=\lim _{n \rightarrow \infty}\left\|y_{n}-T T_{r_{2}}^{f_{2}}\left(y_{n}+\gamma B^{*}\left(A x_{n}-B y_{n}\right)\right)\right\|=0$,

(c) $\left\{x_{n}\right\}_{n=1}^{\infty}$ converges strongly to $p, q \in \Gamma$.

Proof : We set $B_{1}:=A_{f_{1}}$ and $B_{2}:=A_{f_{2}}$. By Lemma 4.4, we know that $B_{1}$ and $B_{2}$ are maximal monotone, $E P\left(f_{1}\right)=B_{1}^{-1} 0, E P\left(f_{2}\right)=B_{2}^{-1} 0, T_{r_{1}}^{f_{1}}=J_{\lambda}^{B_{1}}$ and $T_{r_{2}}^{f_{2}}=J_{\lambda}^{B_{2}}$, so the result is obtained directly by Theorem 3.1 .

\subsection{Split Equality Variational inequality Problem (SEVIP).}

Theorem 4.3. Let $H_{1}$ and $H_{2}$ be Hilbert spaces, $A: H_{1} \rightarrow H_{3}$ and $B: H_{2} \rightarrow H_{3}$ be bounded linear operators, and $S: H_{1} \rightarrow C B\left(H_{1}\right)$ be multivalued Lipschitzian quasi-pseudocontractive self maps of $H_{1}$ and $T: H_{2} \rightarrow C B\left(H_{2}\right)$ be multivalued Lipschitzian quasi-pseudocontractive such that $(I-S)$ and $(I-T)$ are demiclosed at zero. Let $A^{*}$ denotes the adjoint of $A$. Let $B_{1}: H_{1} \rightarrow 2^{H_{1}}$ and $B_{2}: H_{2} \rightarrow 2^{H_{2}}$ be two set valued maximal monotone mappings and $\gamma, \lambda>0$. Given any $x^{*} \in H_{1}, y^{*} \in H_{2}$

(i) if $x^{*}$ and $y^{*}$ are solutions of SEVIP, then $J_{\lambda}^{B_{1}}\left(x^{*}-\gamma A^{*}\left(A x^{*}-B y^{*}\right)\right)=x^{*}$ and $J_{\lambda}^{B_{2}}\left(y_{n}+\right.$ $\left.\gamma B^{*}\left(A x^{*}-B y^{*}\right)\right)=y^{*}$

(ii) Suppose that $J_{\lambda}^{B_{1}}\left(x^{*}-\gamma A^{*}\left(A x^{*}-B y^{*}\right)\right)=x^{*}$ and $J_{\lambda}^{B_{2}}\left(y^{*}+\gamma B^{*}\left(A x^{*}-B y^{*}\right)\right)=y^{*}$, and the solution set of SEVIP are not empty, then $x^{*}$ and $y^{*}$ are solutions of SEVIP.

Proof : We set $B_{1}:=A_{f_{1}}$ and $B_{2}:=A_{f_{2}}$. By Lemma 4.4, we know that $B_{1}$ and $B_{2}$ are maximal monotone, $S=I, T=I$, so the result is obtained directly by Theorem 3.1.

\subsection{Split Equality Optimization Problem (SEOP).}

Theorem 4.4. Let $H_{1}$ and $H_{2}$ be Hilbert spaces. Let $f: H_{1} \rightarrow \mathbb{R}$ and $g: H_{2} \rightarrow \mathbb{R}$ be proper lower semicontinuous convex function of $H$ into $(-\infty,+\infty]$. Let $A: H_{1} \rightarrow H_{3}$ and 
$B: H_{2} \rightarrow H_{3}$ be bounded linear operators, and $S: H_{1} \rightarrow C B\left(H_{1}\right)$ be Lipschitzian quasipseudocontractive and $\mathrm{T}: \mathrm{H}_{2} \rightarrow \mathrm{CB}\left(\mathrm{H}_{2}\right)$ be Lipschitzian quasi-pseudocontractive such that $(I-S)$ and $(I-T)$ are demiclosed at zero. If the solution set of SEOP (95) is nonempty (that is, $\left.\Gamma=\left\{x \in F(S) \cap(\partial f)^{-1} 0, y \in F(T) \cap(\partial g)^{-1} 0: A x_{n}-B y_{n}\right\} \neq \emptyset\right)$. Suppose that $x_{0}, x_{1} \in H_{1}$ and $y_{0}, y_{1} \in H_{2}$ are chosen arbitrarily. Let $\left\{\left(x_{n}, y_{n}\right)\right\}$ be the iterative sequence generated by

$$
\begin{array}{rll}
x_{n+1} & =\beta_{n} x_{0}+\left(1-\beta_{n}\right) u_{n} & \\
u_{n} & =\alpha_{n} x_{n}+\left(1-\alpha_{n}\right) S J_{\lambda}^{\partial f}\left(x_{n}-\gamma A^{*}\left(A x_{n}-B y_{n}\right)\right) & \\
y_{n+1} & =\beta_{n} y_{0}+\left(1-\beta_{n}\right) v_{n} & \\
v_{n} & =\alpha_{n} y_{n}+\left(1-\alpha_{n}\right) T J_{\lambda}^{\partial f}\left(y_{n}+\gamma B^{*}\left(A x_{n}-B y_{n}\right)\right) &
\end{array}
$$

where the parameter $\gamma$ and the sequences $\left\{\alpha_{n}\right\},\left\{\beta_{n}\right\} \subset(0,1)$ satisfying the conditions: (i) $\gamma \in\left(0, \min \left(\frac{2}{\|A\|^{2}}, \frac{2}{\|B\|^{2}}\right)\right),($ ii $) \sum_{n=1}^{\infty} \alpha_{n}<\infty$, (iii) $\lim _{n \rightarrow \infty} \beta_{n}=0$ and $($ iv $) \sum_{n=1}^{\infty} \beta_{n}=\infty$. Then,

(a) $\lim _{n \rightarrow \infty} \Phi_{n}(p, q)$ exists for each $p, q \in \Gamma$,

(b) $\lim _{n \rightarrow \infty}\left\|x_{n}-S J_{\lambda}^{\partial f}\left(x_{n}-\gamma A^{*}\left(A x_{n}-B y_{n}\right)\right)\right\|=\lim _{n \rightarrow \infty}\left\|y_{n}-T J_{\lambda}^{\partial g}\left(y_{n}+\gamma B^{*}\left(A x_{n}-B y_{n}\right)\right)\right\|=0$,

(c) $\left\{x_{n}\right\}_{n=1}^{\infty}$ converges strongly to $p, q \in \Gamma$.

Proof: Set $B_{1}:=\partial f$ and $B_{2}:=\partial g$. Hence the result is obtained directly by Theorem 3.1.

\section{Remark 4.1.}

(1) We obtained strong convergence results from our algorithm without imposing compactness type condition (demi-compactness) on the mapping $S$ and $T$ which appear to a stronger condition.

(2) Chang et al. [14] showed that strong convergence is guaranteed if the maps $S$ and $T$ are semi-compact whereas the condition is not required in our theorem.

(3) The efficiency and implementation of iterative algorithm does not require the calculation or estimation of the operator norms $\|A\|$ and $\|B\|$ which may at times be as difficult as solving the original problem itself.

(4) Our work unify the split equality fixed point problem, split equality null point problem and other related fixed point problems.

(5) The above results for multivalued Lipschizian quasi-pseudo contractive maps are also valid for quasi nonexpansive maps, firmly quasi nonexpansive, demicontractive mappings and hence our results improve and extend many results in the literature [14, 16, 24].

\section{REFERENCES}

[1] A. Aleyner, S. Reich, Block-iterative algorithms for solving convex feasibility problems in Hilbert and in Banach spaces, J. Math. Anal. Appl. 343 (2008) 427-435. https://doi.org/10.1016/j.jmaa.2008.01. 087.

[2] H. Attouch, J. Bolte, P. Redonte, A. Soubeyran, Alternating proximal algorithms for weakly coupled minimization problems. Applications to dynamical games and PDEs. J. Convex Anal. 15 (2008) 485-506.

[3] J.B. BailIon, R.E. Bruck, S. Reich, On the asymptotic behavior of nonexpansive mappings and semigroups in Banach spaces, Houston J. Math. 4 (1978) 1-9.

[4] H.H. Bauschke, P.L. Combettes, A weak-to-strong convergence principle for Fejér-Monotone methods in Hilbert spaces, Math. Oper. Res. 26 (2001) 248-264. https://doi.org/10.1287/moor . 26.2 . 248.10558.

[5] E. Blum, W. Oettli, From optimization and variational inequalities to equilibrium problems. Math. Stud. 63 (1994). 123-145. 
[6] O.A. Boikanyo, H. Zegeye, The split equality fixed point problem for quasi-pseudo-contractive mappings without prior knowledge of norms, Numer. Funct. Anal. Optim. 41 (2020) 759-777. https://doi.org/10. 1080/01630563.2019.1675170.

[7] C. Byrne, Iterative oblique projection onto convex subsets and the split feasibility problem, Inverse Probl. 18 (2002) 441-453.

[8] Y. Censor, T. Bortfeld, B. Martin, A. Trofimov, A unified approach for inversion problems in intensity-modulated radiation therapy, Phys. Med. Biol. 51 (2006) 2353-2365. https://doi .org/10.1088/ 0031-9155/51/10/001.

[9] Y. Censor, T. Elfving, A multiprojection algorithm using Bregman projections in a product space, Numer Algor. 8 (1994) 221-239. https://doi.org/10.1007/BF02142692.

[10] Y. Censor, T. Elfving, N. Kopf, T. Bortfeld, The multiple-sets split feasibility problem and its applications. Inverse Probl. 21 (2005) 2071-2084.

[11] Y. Censor, A. Motova, A. Segal, Perturbed projections and subgradient projections for the multiple-sets split feasibility problem, J. Math. Anal. Appl. 327 (2007) 1244-1256. https://doi.org/10.1016/j.jmaa. 2006.05.010.

[12] S. Chang, R.P. Agarwal, Strong convergence theorems of general split equality problems for quasi-nonexpansive mappings, J. Inequal. Appl. 2014 (2014) 367. https://doi.org/10.1186/ 1029-242X-2014-367.

[13] S. Chang, L. Wang, Y.K. Tang, G. Wang, Moudafi's open question and simultaneous iterative algorithm for general split equality variational inclusion problems and general split equality optimization problems, Fixed Point Theory Appl. 2014 (2014) 215. https://doi.org/10.1186/1687-1812-2014-215.

[14] S. Chang, L. Wang, L.-J. Qin, Split equality fixed point problem for quasi-pseudo-contractive mappings with applications, Fixed Point Theory Appl. 2015 (2015) 208. https://doi .org/10.1186/s13663-015-0458-3.

[15] A. Cegielski, Iterative methods for fixed point problems in hilbert spaces, Lecture Notes in Mathematics, Springer, Heidelberg (2012).

[16] H. Che, M. Li, A simultaneous iterative method for split equality problems of two finite families of strictly pseudononspreading mappings without prior knowledge of operator norms, Fixed Point Theory Appl. 2015 (2015) 1. https://doi.org/10.1186/1687-1812-2015-1.

[17] C.E. Chidume, C.O. Chidume, N. Djitté, M.S. Minjibir, Convergence theorems for fixed points of multivalued strictly pseudocontractive mappings in Hilbert spaces, Abstr. Appl. Anal. 2013 (2013) 629468. https://doi.org/10.1155/2013/629468.

[18] C.E. Chidume, A.U. Bello, P. Ndambomve, Strong and $\triangle$-convergence theorems for common fixed points of a finite family of multivalued demicontractive mappings in CAT(0) spaces, Abstr. Appl. Anal. 2014 (2014) 805168. https://doi.org/10.1155/2014/805168.

[19] P.L. Combettes, S.A. Hirstoaga, Equilibrium programming in Hilbert spaces. J. Nonlinear Convex Anal. 6 (2005) 117-136.

[20] F.O. Isiogugu, M.O. Osilike, Convergence theorems for new classes of multivalued hemicontractive-type mappings, Fixed Point Theory Appl. 2014 (2014) 93. https://doi.org/10.1186/1687-1812-2014-93.

[21] P. Jailoka, S. Suantai, Split common fixed point and null point problems for demicontractive operators in Hilbert spaces, Optimization Methods and Software. 34 (2019) 248-263. https://doi.org/10.1080/ 10556788.2017.1359265.

[22] E. Masad, S. Reich, A note on the multiple-set split convex feasibility problem in Hilbert spaces, J. Nonlinear Convex Anal. 8 (2007) 367-371.

[23] A. Moudafi, A relaxed alternating CQ-algorithm for convex feasibility problems, Nonlinear Anal.: Theory Meth. Appl. 79 (2013) 117-121. https://doi.org/10.1016/j.na.2012.11.013.

[24] A. Moudafi, Split monotone variational inclusions, J. Optim. Theory Appl. 150 (2011) 275-283. https: //doi.org/10.1007/s10957-011-9814-6.

[25] A. Moudafi, E. Al-Shemas, Simultaneous iterative methods forsplit equality problem, Trans. Math. Program. Appl. 1 (2013) 1-11. 
[26] M.O. Osilike, D.I. Igbokwe, Weak and strong convergence theorems for fixed points of pseudocontractions and solutions of monotone type operator equations, Computers Math. Appl. 40 (2000) 559-567. https: //doi.org/10.1016/S0898-1221(00)00179-6.

[27] R. Rockafellar, On the maximal monotonicity of subdifferential mappings, Pac. J. Math. 33 (1970) 209-216. https://doi.org/10.2140/pjm.1970.33.209.

[28] Y.J. Cho, Some notes on Ishikawa iteration for multi-valued mappings, Bull. Korean Math. Soc. 48 (2011) 575-584. https://doi.org/10.4134/BKMS.2011.48.3.575.

[29] S. Takahashi, W. Takahashi, M. Toyoda, Strong Convergence Theorems for Maximal Monotone Operators with Nonlinear Mappings in Hilbert Spaces, J. Optim. Theory Appl. 147 (2010) 27-41. https://doi.org/ 10.1007/s10957-010-9713-2.

[30] H.-K. Xu, Inequalities in Banach spaces with applications, Nonlinear Anal.: Theory Meth. Appl. 16 (1991) 1127-1138. https://doi.org/10.1016/0362-546X (91)90200-K.

[31] H.-K. Xu, A variable Krasnosel'skii-Mann algorithm and the multiple-set split feasibility problem, Inverse Probl. 22 (2006) 2021-2034. https://doi.org/10.1088/0266-5611/22/6/007.

[32] H.-K. Xu, Iterative methods for the split feasibility problem in infinite-dimensional Hilbert spaces, Inverse Probl. 26 (2010) 105018. https://doi.org/10.1088/0266-5611/26/10/105018.

[33] Q. Yang, The relaxed CQ algorithm solving the split feasibility problem, Inverse Probl. 20 (2004) 1261-1266. https://doi.org/10.1088/0266-5611/20/4/014.

[34] Y. Yao, R. Chen, G. Marino, Y.C. Liou, Applications of fixed-point and optimization methods to the multiple-set split feasibility problem, J. Appl. Math. 2012 (2012) 927530. https://doi.org/10.1155/ 2012/927530.

[35] Y. Yao, Y.-C. Liou, J.-C. Yao, Split common fixed point problem for two quasi-pseudo-contractive operators and its algorithm construction, Fixed Point Theory Appl. 2015 (2015) 127. https://doi.org/10.1186/ s13663-015-0376-4.

[36] J. Zhao, Q. Yang, Several solution methods for the split feasibility problem, Inverse Probl. 21 (2005) 1791-1799. https://doi.org/10.1088/0266-5611/21/5/017.

[37] H. Zhou, Strong convergence of an explicit iterative algorithm for continuous pseudo-contractions in Banach spaces, Nonlinear Anal.: Theory Meth. Appl. 70 (2009) 4039-4046. https://doi.org/10.1016/j.na. 2008.08 .012$. 